\section{(2) OPEN ACCESS}

\title{
RNA-binding protein RALY reprogrammes mitochondrial metabolism via mediating miRNA processing in colorectal cancer
}

\author{
Lei Sun, ${ }^{1}$ Arabella Wan, ${ }^{2}$ Zhuolong Zhou, ${ }^{3}$ Dongshi Chen, ${ }^{4}$ Heng Liang, ${ }_{1}{ }^{2}$ Chuwei Liu, ${ }^{2}$ \\ Shijia Yan, ${ }^{1}$ Yi Niu, ${ }^{1}$ Ziyou Lin, ${ }^{1}$ Siyue Zhan, ${ }^{1}$ Shanfeng Wang, ${ }^{5}$ Xianzhang Bu, ${ }^{1}$ \\ Weiling $\mathrm{He}^{2,6}$ Xiongbin Lu (1), ${ }^{3,7}$ Anlong $\mathrm{Xu}$ (1) ${ }^{8,9}$ Guohui Wan (1) ${ }^{1}$
}

For numbered affiliations see end of article.

\section{Correspondence to}

Professor Guohui Wan, School of Pharmaceutical Sciences, Sun Yat-Sen University, Guangzhou, China;

wanguoh@mail.sysu.edu.cn, Professor Anlong Xu, School of Life Sciences, Beijing University of Chinese Medicine, Beijing,

China;

xuanlong@bucm.edu.cn and Professor Xiongbin Lu, Department of Medical and Molecular Genetics, Indiana University School of Medicine, Indianapolis, IN, USA; xiolu@iu.edu

LS and AW contributed equally.

LS and AW are joint first authors.

Received 10 January 2020 Revised 8 October 2020 Accepted 27 October 2020 Published Online First 20 November 2020

\section{Check for updates}

(C) Author(s) (or their employer(s)) 2021. Re-use permitted under CC BY-NC. No commercial re-use. See rights and permissions. Published by BMJ.

To cite: Sun $L$, Wan $A$ Zhou Z, et al. Gut

2021:70:1698-1712.

\section{ABSTRACT}

Objective Dysregulated cellular metabolism is a distinct hallmark of human colorectal cancer (CRC). However, metabolic programme rewiring during tumour progression has yet to be fully understood.

Design We analysed altered gene signatures during colorectal tumour progression, and used a complex of molecular and metabolic assays to study the regulation of metabolism in CRC cell lines, human patient-derived xenograft mouse models and tumour organoid models. Results We identified a novel RNA-binding protein, RALY (also known as hnRNPCL2), that is highly associated with colorectal tumour aggressiveness. RALY acts as a key regulatory component in the Drosha complex, and promotes the post-transcriptional processing of a specific subset of miRNAs (miR-483, miR-676 and miR-877). These miRNAs systematically downregulate the expression of the metabolismassociated genes (ATP5I, ATP5G1, ATP5G3 and CYC1) and thereby reprogramme mitochondrial metabolism in the cancer cell. Analysis of The Cancer Genome Atlas (TCGA) reveals that increased levels of RALY are associated with poor prognosis in the patients with CRC expressing low levels of mitochondrion-associated genes. Mechanistically, induced processing of these miRNAs is facilitated by their N6-methyladenosine switch under reactive oxygen species (ROS) stress. Inhibition of the $m^{6} A$ methylation abolishes the RALY recognition of the terminal loop of the pri-miRNAs. Knockdown of RALY inhibits colorectal tumour growth and progression in vivo and in organoid models.

Conclusions Collectively, our results reveal a critical metabolism-centric role of RALY in tumour progression, which may lead to cancer therapeutics targeting RALY for treating CRC.

\section{INTRODUCTION}

The acquired capability of unrestrained replicative potential has been a representative hallmark for cancer cells. ${ }^{1}$ To meet the requirement for hyperproliferation, cancer cells shift intracellular energy metabolism and mainly rely on glycolysis pathways instead of mitochondrial oxidative phosphorylation (OXPHOS) to support their energy demand and biomass synthesis, even when the oxygen level is sufficient. ${ }^{12}$ Dysregulation of metabolism in cancer

\section{Significance of this study}

What is already known on this subject?

- Dysregulation of metabolism in cancer cells is dynamically regulated at many cancer hallmarks.

- Metabolic reprogramming in cancer cells could be impacted by post-translational events and epigenetic regulation of genes.

- RALY was identified in the spliceosome complex and in the Drosha microprocessor complex.

- The $\mathrm{m}^{6} \mathrm{~A}$ modification is found to alter RNA structure to enhance HNRNPC binding, and HNRNPA2B1 could bind to the $\mathrm{m}^{6} \mathrm{~A}$ marks in some pri-miRNA transcripts to promote their processing.

What are the new findings?

- RALY is highly associated with metastasis of malignant colorectal cancer (CRC).

- RALY acts as a key regulatory component in the Drosha complex, and promotes the posttranscriptional processing of a specific subset of miRNAs.

- RALY-mediated miRNAs processing is modulated by N6-methyladenosine switch, and reprogrammes mitochondrial metabolism by downregulating the electron transport chain genes.

- Inhibition of RALY suppresses colorectal tumour growth and progression in vivo and in the organoid models.

How might it impact on clinical practice in the foreseeable future?

- Our report first reveals a critical metabolismcentric role of RALY in tumour progression. It provides a novel therapeutic strategy to target RALY for CRC treatment.

cells is actively regulated at many cancer hallmarks, including proliferation, angiogenesis, invasion and metastasis. ${ }^{3}$ In a recent study, we reported that USP13 as a key regulator rewires energy metabolism through deubiquitinating ATP citrate synthase (ACLY) and 2-oxoglutarate dehydrogenase (OGDH) and promotes ovarian tumour progression. ${ }^{5}$ 
Emerging evidence has shown that metabolic reprogramming in cancer cells could be impacted by post-translational events and epigenetic modulation of gene expression. ${ }^{6-8}$ Identification of key regulators in mediating cancer cell metabolism could lead to a new therapeutic approach for cancer treatment.

As 'energy factories', mitochondria control the bioenergetics, biosynthetic and signalling organelles of cancer cells and serve as an attractive target for cancer therapy. ${ }^{9-11}$ Previous reports showed that inhibition of electron transport chain (ETC) genes could sensitise cancer cells to glucose depletion and reduce tumour progression. ${ }^{12}{ }^{13}$ However, regulation of ETC gene expression in tumour progression remains largely unknown. Recent studies indicate that epigenetic regulation has an essential role in controlling cellular signalling pathways during tumourigenesis. ${ }^{1415}$ Delineation of multifaceted epigenetic changes in mitochondrial metabolism during tumour progression would provide insights into the crucial mechanism by which cancer cells adapt to a changing microenvironment.

Recently, miRNAs have been found to act as master gene regulators, similar to transcription factors, to mediate gene expression patterns in various cellular activities, including cell metabolism. ${ }^{16} 17$ C-Myc suppression of miR-23a and miR$23 \mathrm{~b}$ enhances mitochondrial glutaminase expression, leading to elevated glutamine catabolism in prostate cancer and B lymphoma. ${ }^{18}$ The RNA binding protein Lin 28 inhibits let- 7 biogenesis to promote the insulin-PI3K-mTOR pathway and increase glucose uptake. ${ }^{19}$ MiR-103, miR-107 and miR-143 are involved in regulating insulin-mediated glucose metabolism, ${ }^{20} 21$ while miR-33 cooperates with SREBPs to target ABCA1 and coordinate cholesterol biosynthesis and trafficking. ${ }^{22}$ MiRNAs have been known to provide a functional link between metabolic reprogramming and tumourigenesis. In this study, we identify a novel RNA-binding protein, RALY (HnRNP-Associated with Lethal Yellow, also called hnRNPCL2), as a crucial indicator for tumour progression in colorectal cancer (CRC). We present here a role of RALY in reprogramming mitochondrial metabolism via mediating miRNA processing in CRC, and highlight the potential therapeutic strategy by targeting RALY in CRC therapy.

\section{Materials and methods}

\section{RNA immunoprecipitation}

As previously described, ${ }^{23}$ RNA immunoprecipitation (RIP) assay was performed using the Magna RIP RNA-Binding Protein Immunoprecipitation Kit (17-700, Merck Millipore, USA) in accordance with manufacturer's protocol. Stable RALY-overexpressed HEK293T cells were collected and total RNA was obtained by re-suspending and dispersing the cell pellet in complete RIP Lysis Buffer. Antibodies of RALY (ab170105, Abcam) or negative control normal rabbit IgG (PP64B) in magnetic beads were added to pull down specific RNAs. Enriched microRNAs were subject to high-throughput NGS sequencing and validated by quantitative RT-PCR.

\section{Metabolic detection}

ECAR and oxygen consumption rate (OCR) were monitored using a Seahorse Bioscience Analyzer (XF96) according to the manufacturer's instructions. Cells were seeded in 96-well plates at a density of $5-8 \times 10^{3}$ cells per well with growth medium for at least 18 hours. Next, the medium was changed to base medium (DMEM, $143 \mathrm{mM} \mathrm{NaCl}$, phenol red, $\mathrm{pH}$ 7.35). For ECAR analysis, cells were added with the medium containing $2 \mathrm{mM}$ glutamine ( $\mathrm{pH} 7.35$ ) and monitored every $3 \mathrm{~min}$ following successive administration of $10 \mathrm{mM}$ of glucose, and inhibitors $(1 \mu \mathrm{M}$ oligomycin and $50 \mathrm{mM}$ 2-deoxyglucose). For OCR analysis, cells were added with Mito stress-test base medium $(10 \mathrm{mM}$ glucose, $2 \mathrm{mM}$ glutamine, $1 \mathrm{mM}$ pyruvate, $\mathrm{pH} \mathrm{7.4)}$ and monitored every $3 \mathrm{~min}$ following successive administration of inhibitors $(2 \mu \mathrm{M}$ oligomycin, $2 \mu \mathrm{M}$ FCCP, or $0.5 \mu \mathrm{M}$ rotenone/antimycin A). ${ }^{11}$

A detailed description of the materials and methods can be found in online supplemental materials and methods.

\section{RESULTS \\ Upregulation of RALY is associated with aggressiveness in CRC}

Specific focal gene amplifications, gene losses and altered geneexpression levels have been identified as signature events for CRC progression. ${ }^{24}$ We conducted intensive analysis of gene alterations in CRC from the TCGA database by using genomic set enrichment analysis (GSEA), and identified a specific group of genes related to RNA alternative splicing that is highly associated with tumour metastasis (figure 1A). Among those genes, a novel RNA binding protein, RALY was identified as a molecular signature associated with tumour aggressiveness from the integrative analysis of tumour stages, lymph node invasion, vascular invasion and distant metastasis status at the time of surgery (figure 1B). Amplification of the RALY gene occurs frequently in human cancers and locates in the proximity of E2F1 (figure 1C and D). Upregulation of RALY is found in 56\% of CRC cases (figure 1E). We demonstrate that RALY is overexpressed in nine human $\mathrm{CRC}$ cell lines as compared with the normal human colon epithelial cell line (figure $1 \mathrm{~F}-\mathrm{H}$ ). By using CRC patient tissue samples locally procured, we further validated the upregulation of RALY in the CRC tumours in comparison with their adjacent normal tissues (figure $1 \mathrm{I}$ and $\mathrm{J}$ and online supplemental figure 1) and (online supplemental table 1). Analysis of TCGA database and the local CRC database revealed that expression of RALY is tightly associated with its gene copy number (figure $1 \mathrm{~K}$ ) and high expression of RALY correlates with poor clinical outcome in CRC patients (figure 1L and online supplemental figure 2A). By correlation analysis with clinical data from TCGA (colon adenocarcinoma (COAD) + rectum adenocarcinoma (READ)), the expression of RALY is significantly correlated with several univariate clinical factors including tumour stage and distant metastasis, but not with the status of KRAS, BRAF and MSI, indicating that RALY may be used as an independent prognostic biomarker for CRC metastasis (online supplemental figure 2B) and (online supplemental table 2).

\section{RALY interacts with the Drosha complex and regulates a subset of miRNA expression}

Previously microRNA biogenesis has been shown to coordinate with alternative splicing under stress in the supraspliceosome, ${ }^{2526}$ and RALY was identified in the spliceosome complex ${ }^{27}$ and in the Drosha microprocessor complex. ${ }^{28}$ However, the functional role of RALY in microRNA biogenesis remains unknown. We examined the physical interaction of RALY with the Drosha microprocessor (figure 2A). Endogenous RALY was validated in the Drosha immunoprecipitate, and conversely, Drosha and DGCR8 were detected in the RALY immunoprecipitate. We also verified the colocalisation of RALY and Drosha in the nucleus (online supplemental figure $2 \mathrm{C}$ ). To examine the effect of RALY on miRNA biogenesis, we performed the analysis of global mRNA and miRNA expression by next-generation sequencing in the RALY-knockdown HCT116 cells, and by RALY RIP sequencing in the RALY-overexpressed $293 \mathrm{~T}$ cells (figure 2B and online supplemental table 3). Depletion of RALY 
A

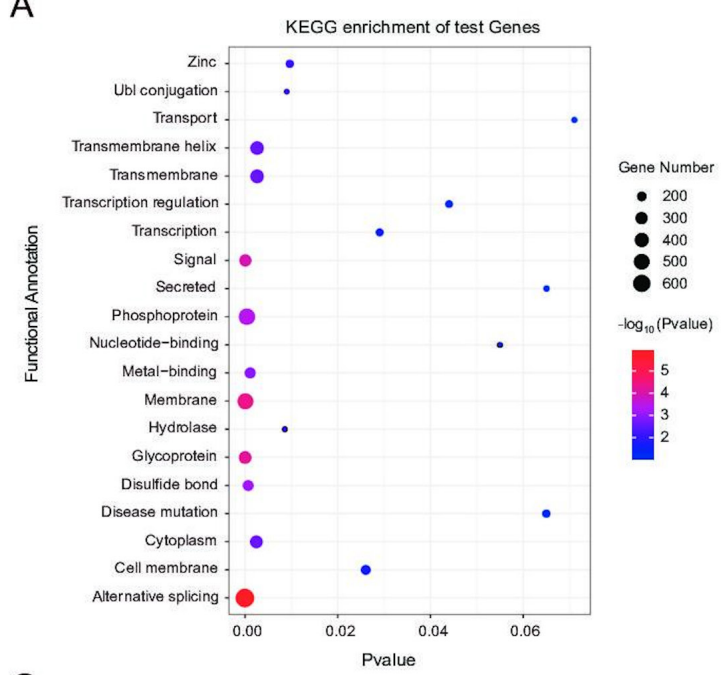

C

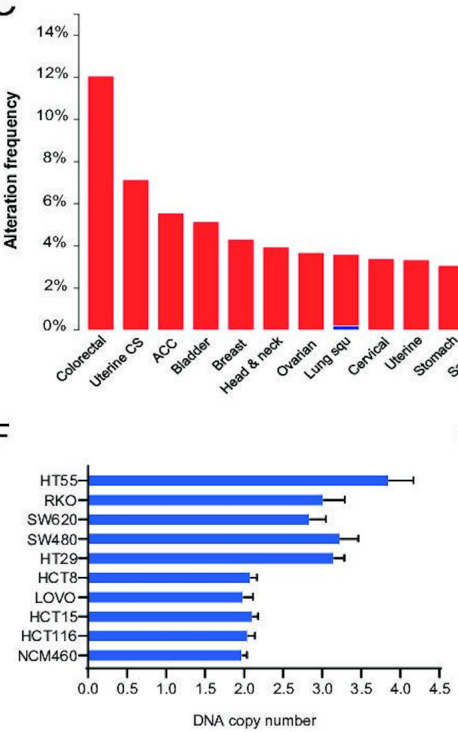

I

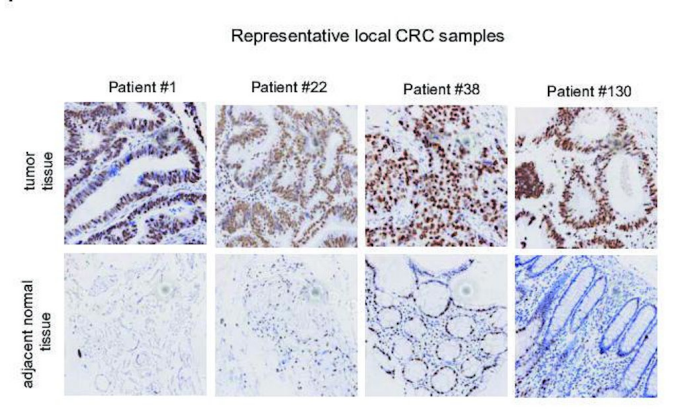

J
B

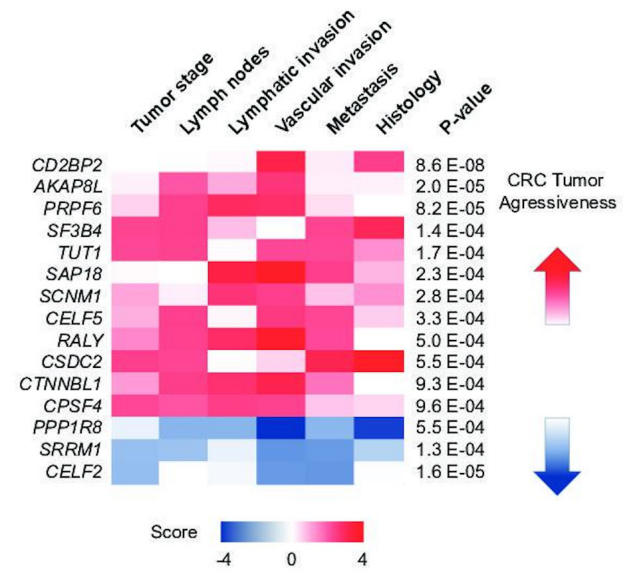

D

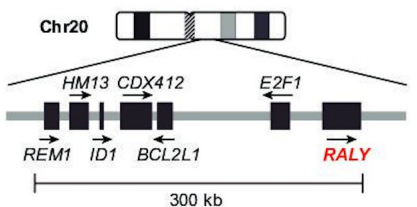

E

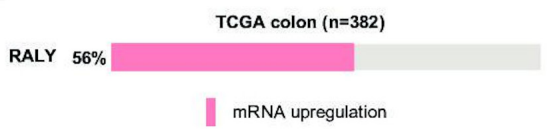

$\mathrm{H}$
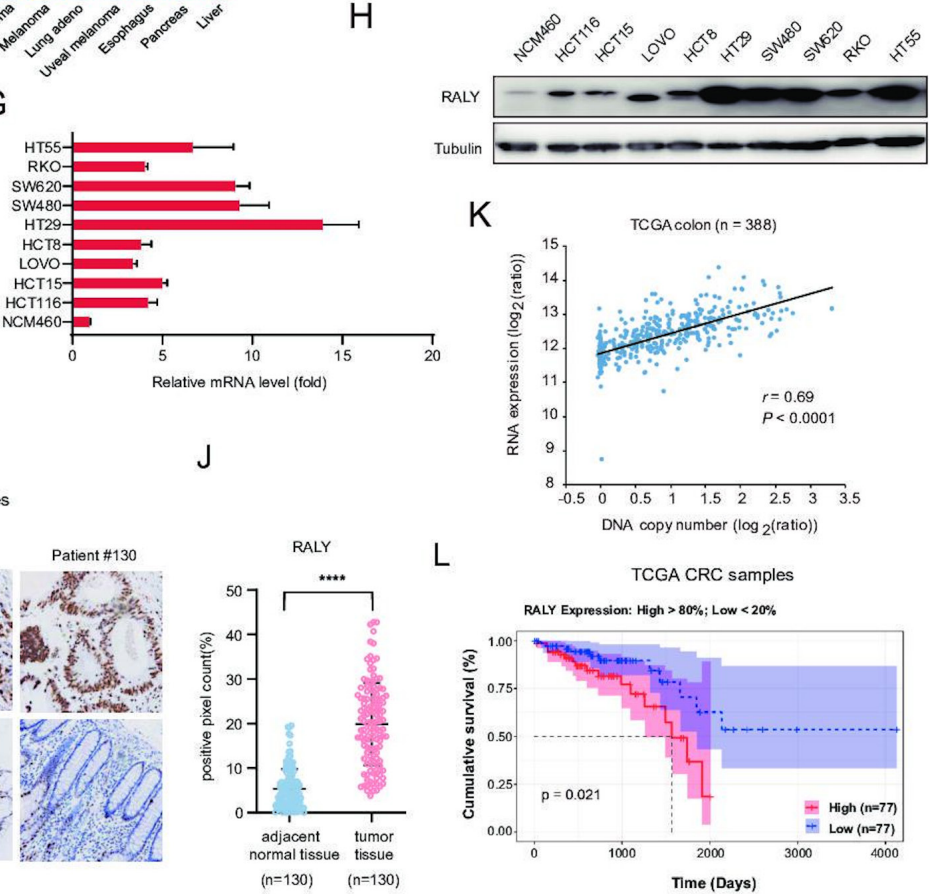

L
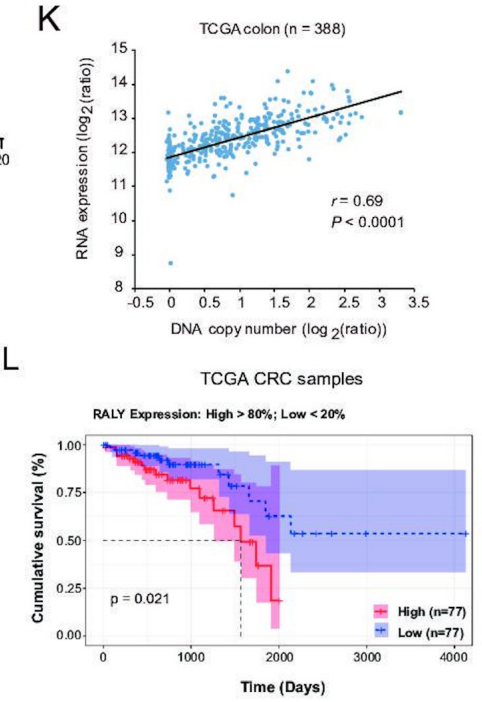

Figure 1 Upregulation of RALY is associated with aggressiveness in colorectal cancer. (A) Functional annotation of altered expression of genes that are associated with CRC tumour aggression. (B) Gene-expression signatures involved in RNA alternative splicing are associated with CRC tumour aggression. Tumour aggressiveness is defined according to selected clinical assays that are shown in colour, with red indicating markers of tumour aggressiveness and blue indicating markers of less-aggressiveness tumours. (C) Amplification of the RALY gene occurs frequently in human cancers. (D) Schematic diagram of amplified genes adjacent to RALY in the human genome. (E) Upregulation of RALY is frequently found in CRC patients. $(F-H)$ Copy numbers (F), relative mRNA expression levels (G) and protein expression levels $(\mathrm{H})$ of RALY in human CRC cell lines. NCM460 is a normal human colon epithelial cell line as a control. (I) RALY expression level in representative local clinical CRC patients and their adjacent normal tissues. (J) Quantification of RALY upregulation in 130 local CRC tumours compared with adjacent normal tissues from IHC results. (K) Scatter plots of RALY copy number vs mRNA expression in TCGA database. Pearson correlation coefficients $(r)$ and $p$ values are shown. (L) Upregulation of RALY is significantly associated with poorer overall survival rates in CRC patients. Data information: in all relevant panels, ${ }^{* * * *} p<0.0001$; two-tailed t-test. Data are presented as mean \pm SD and are representative of three independent experiments. CRC, colorectal cancer; IHC, immunohistochemistry; TCGA, The Cancer Genome Atlas. 
A

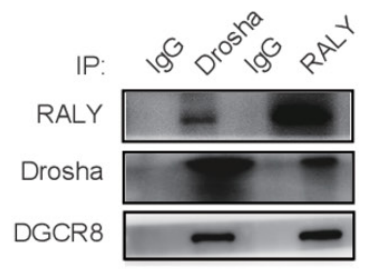

WCL:

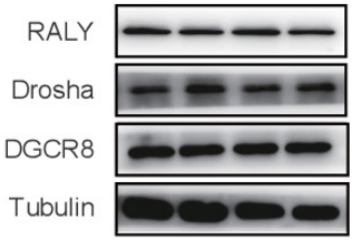

E
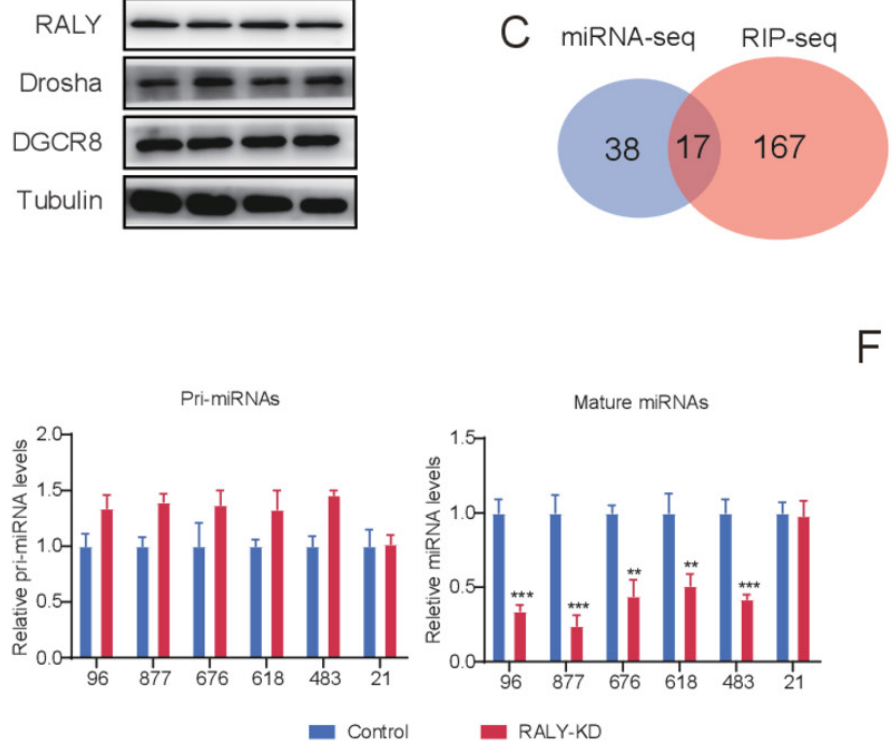

F
D

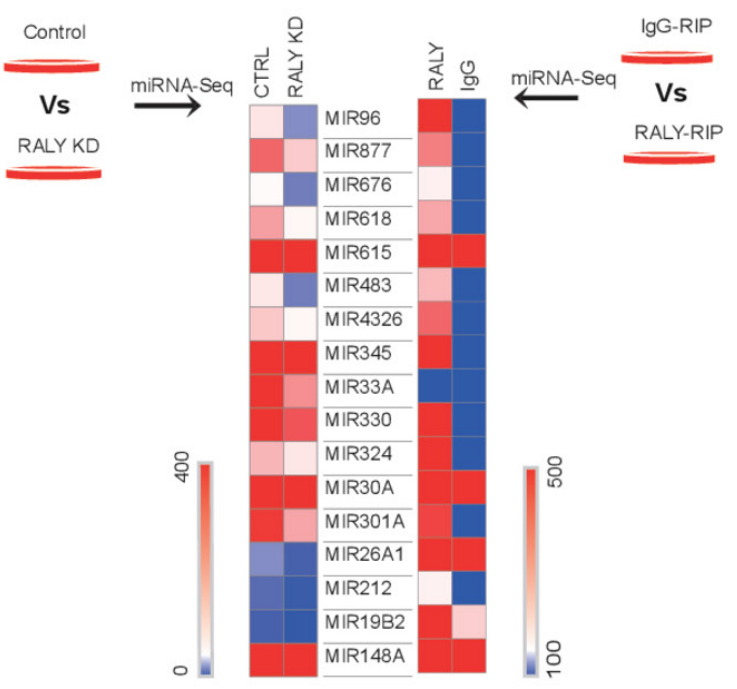

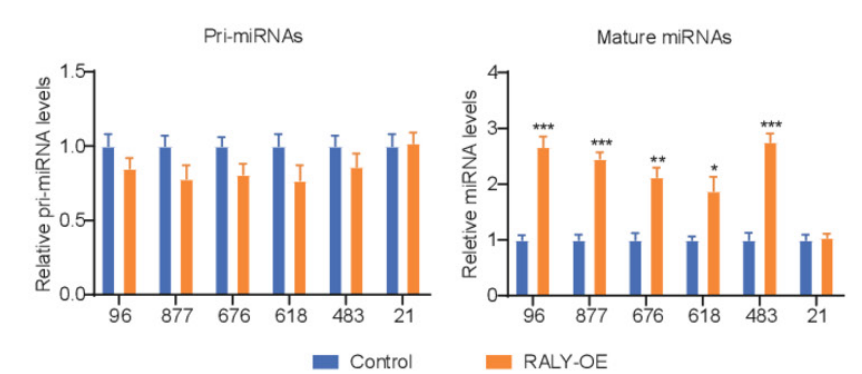

G
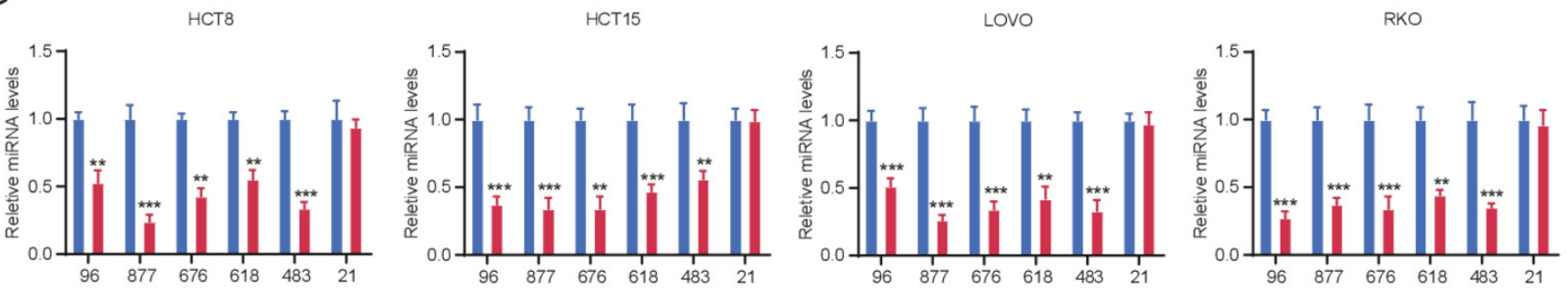

HT29
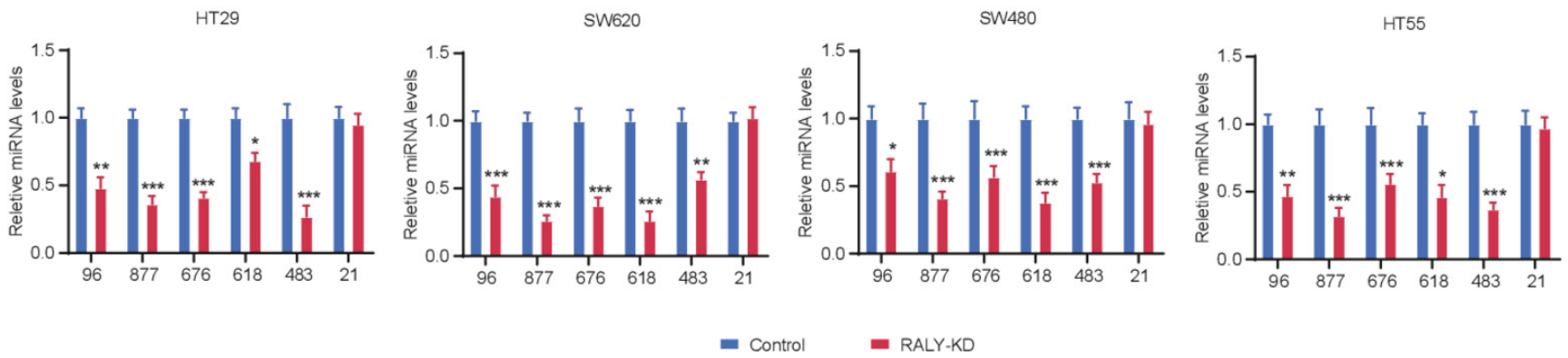

Figure 2 RALY interacts with the Drosha complex and regulates a subset of miRNA expression. (A) Interaction between endogenous RALY and Drosha/DGCR8 complex. Immunoprecipitation (IP) and Western blot analysis were performed using indicated antibodies. WCL, whole-cell lysate. (B) Pipeline for identifying relevant RALY-dependent genes and miRNAs in CRC. (C) Venn diagram showing the overlap of miRNA-seq results and RIP-seq results. RIP-seq identified differentially expressed miRNAs interacting with RALY antibody in the 293T cell line compared with lgG. (fold change $>4$ ) (D) Depletion of RALY inhibited expression of a subset of miRNAs. Total RNAs from control and RALY knockdown (KD) were subject to global miRNAprofiling analysis using RNA sequencing. Colour intensity refers to miRNA expression level. (E-F) Levels of primary form and mature form of the RALYdependent miRNA in RALY-KD (E) and RALY-overexpression (F) HCT116 cells. (G) Validation of RALY-dependent miRNAs expression in human CRC cell lines with RALY-KD. Data information: in all relevant panels, ${ }^{*} \mathrm{p}<0.05 ;{ }^{*} \mathrm{p}<0.01$; ${ }^{* *} \mathrm{p}<0.001$; two-tailed t-test. Data are presented as mean $\pm \mathrm{SD}$ and are representative of three independent experiments. CRC, colorectal cancer; RIP, RNA immunoprecipitation. 
significantly decreased the expression levels of a subset of 17 miRNAs (cutoff $>2$ fold), while overexpression of RALY significantly increased their level enrichment in the RALY immunoprecipitate complex (figure $2 \mathrm{C}$ and $\mathrm{D}$ ). To determine whether RALY regulates the miRNA expression at transcriptional or post-transcriptional levels, we detected the levels of both primiRNAs and mature miRNAs in the RALY-knockdown or RALYoverexpression HCT116 cells. As a control, miR-21 was used in the assay as it does not depend on the RALY expression level. No notable differences were detected on the transcription of pri-miRNAs including pri-miR-96, pri-miR-483, pri-miR-618, pri-miR-676 and pri-miR-877 in the control cells and RALYknockdown or RALY-overexpression cells. However, levels of mature forms of the RALY-dependent miRNA, but not control miR-21, were dramatically decreased when RALY was knocked down in HCT116 cells. Conversely, these RALY-dependent miRNAs were elevated in the RALY-overexpression HCT116 cells (figure 2E,F). To validate the universal RALY-dependency of these miRNAs in CRC, we further analysed the mature forms of these miRNAs in eight more CRC cell lines, including HCT8, HCT15, LOVO, RKO, HT29, SW620, SW480 and HT55 cell lines. Consistently, knockdown of RALY significantly suppressed the levels of RALY-dependent miRNAs, but not the level of the control miR-21, in these cell lines (figure 2G). These results indicate that RALY promotes the expression of specific miRNAs at the post-transcriptional level.

\section{RALY reprogrammes mitochondrial metabolism in CRC through mediating miRNA expression}

To investigate the functional role of RALY in CRC tumour progression, we establish stable RALY-knockdown models in HCT116, HCT15 and LOVO cells with two independent shRNAs (shRALY\#1 and \#2) (online supplemental figure 3A). To determine the biological function of RALY, we performed GSEA in the RALY-knockdown HCT116 cells, and found that the OXPHOS pathways including energy metabolism and mitochondrial respiratory ETC are significantly enriched in the RALY-depleted cells (figures $2 \mathrm{~B}$ and $3 \mathrm{~A}$ ). Knockdown of RALY induced the expression of some mitochondrion-related ETC genes, including ATP5I, ATP5G1, ATP5G3, ATP5J2 and CYC1 at both RNA and protein levels (figure 3B and C). We reasoned that knockdown of RALY reprograms mitochondrial metabolism in CRC, and thereby limits the synthesis of intermediates in the TCA cycle from the OXPHOS pathways. Marked reduction of extracellular acidification rate (ECAR) levels was observed in the RALY-knockdown cells, indicating the influence of RALY on aerobic glycolysis, while increased basal OCR levels in the RALY-knockdown cells suggested a role of RALY in aerobic respiration. Conversely, overexpression of RALY elevated the ECAR levels and decreased the OCR levels (figure 3D and E and online supplemental figure 3B-F). Significant reduction of ATP levels and induction of ROS levels were also observed in the RALY-knockdown cells and the opposites were seen in the RALY-overexpressed cells (figure $3 \mathrm{~F}$ and $\mathrm{G}$ and online supplemental figure $3 \mathrm{GH}$ ). As a rapid turnover of $\mathrm{NAD}+$ is required in cancer cells due to their elevated metabolic needs, ${ }^{29}$ depletion of RALY significant increased the $\mathrm{NAD}+$ levels and the NAD+/NADH ratios, indicating reduced reductive flux (figure $3 \mathrm{H}$ and online supplemental figure 3I,J). In line with our hypothesis, we find that knockdown of RALY significantly increased the expression of ETC genes, leading to reduced ATP production, induced ROS level and the NAD+/ NADH ratio in CRC cells.
To validate the effects of RALY on mitochondrial metabolism are mainly mediated by mitochondrion-related ETC genes, we have constructed stable knockdown CRC cells (ATP5I-KD, ATP5G1-KD, ATP5G3-KD and CYC1-KD) by lentiviral shRNAs (figure 3I and online supplemental figure 4A,B). Knockdown of ATP5I, ATP5G1, ATP5G3 and CYC1 significantly reduced ROS levels in the RALY-depleted CRC cells (figure 3J and supplemental figure $4 \mathrm{C}$ ). To determine how RALY regulates those mitochondrion-related ETC genes, we conducted miRNA-target prediction by using comprehensive tools including TargetScan, MiRanda and miRBase, and identified six specific binding sites at the 3' untranslated region (3'UTR) of those ETC genes for the RALY-dependent miRNAs including miR-483, miR-676 and miR-877 (figure 2B and supplemental figure 4D). To prove the direct effects of these miRNAs on ETC gene expression, we cloned the ETC genes' 3'UTR sequences into a dual luciferase reporter construct and generated deletion mutant vectors (online supplemental figure 4DE). Overexpression of miR-483, miR-676 and miR-877 reduced the relative luciferase activity of the targeted ETC 3'UTRs while their specific inhibitors (antagomiRs) increased the luciferase activity signals. Deletion mutants on the miRNA-binding sites blocked the regulatory effects mediated by these miRNAs (online supplemental figure 4F). Consistently, overexpression of miR-483, miR-676 and miR-877 downregulated the expression of the target ETC genes at both RNA and protein levels (figure $3 \mathrm{~K}$ and $\mathrm{L}$ and online supplemental figure 5A-D). Conversely, overexpression of miR-483, miR-676 and miR-877 rescued the effects on mitochondrial metabolism caused by RALY depletion in CRC cells (figure 3M and supplemental figure 5E-L). These results illustrate that RALY modulates mitochondrial metabolism through mediating miRNA expression.

\section{RALY regulates CRC cell proliferation and apoptosis under ROS stress}

Mitochondrial metabolism is a key role in cellular energy, biosynthesis and cell growth, and ROS is controlled by mitochondria. It has been shown to affect the tumourigenic and metastatic phenotypes. ${ }^{30}$ Since our results indicate that RALY regulates ROS production by mediating miRNA processing, we postulated that RALY may participate in mitochondrial ROS-involved stress signalling and thus affect tumour growth. Knockdown of RALY remarkably suppressed CRC cell proliferation as indicated by decreasing cell numbers (figure $4 \mathrm{~A}$ and $\mathrm{B}$ and online supplemental figure 6AB), reducing EDU staining (figure $4 \mathrm{E}$ and $\mathrm{F}$ ) and fewer cellular survival dots (figure 4G). To determine whether the mitochondrion-mediated ROS production by RALY is crucial for cell growth in CRC, we use a ROS scavenger, $\mathrm{N}$-acetyl L-cysteine (NAC), to deplete the ROS level in cancer cells. Treatments of NAC partially rescued cancer cell growth in the RALY-knockdown cells, but the rescue effect was not dependent on NAC concentrations (figure 4C, E and G). Similarly, overexpression of ETC genes-related miRNAs (miR483, miR-676 and miR-877) restored cell growth and proliferation in the RALY-knockdown cells (figure 4D, F and G). We next examined the function of RALY in cell survival by measuring the levels of Ki67 and caspase-3. RALY depletion induced significant apoptosis (indicated by cleaved caspase-3) by flow cytometry. Treatment of NAC or overexpression of the miRNAs related to ETC genes restored cell survival in the RALY-knockdown cells (figure 4H1), as validated by reduction of the ROS level in these rescue of cells compared with the RALY-knockdown cells (figure $4 \mathrm{~J}$ ). These results suggest that RALY plays a crucial role in 
A

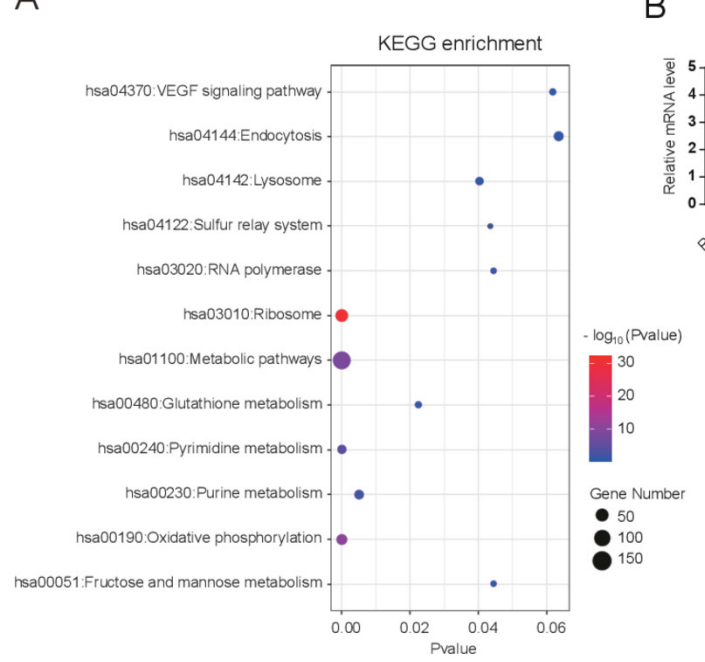

D

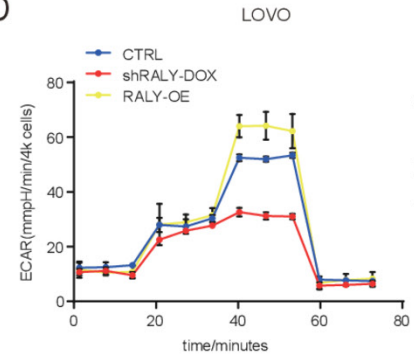

F

Lovo G

B

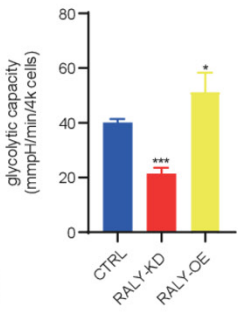

$\mathrm{H}$

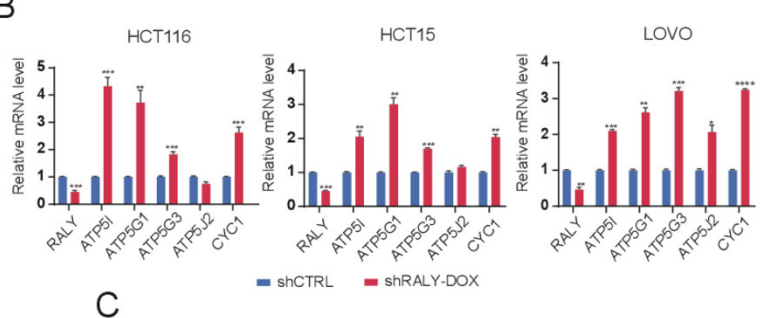

C
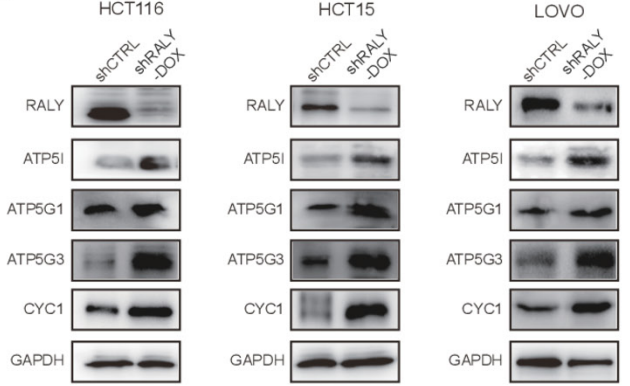

E
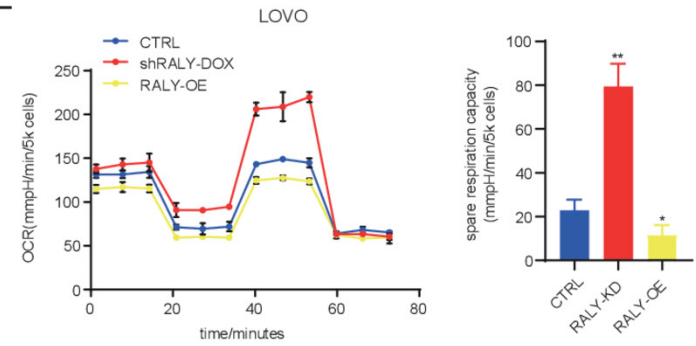

I

LOVO

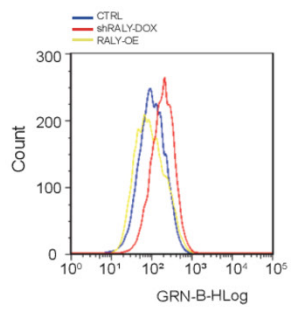

K

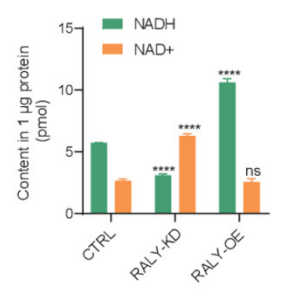

LOVO
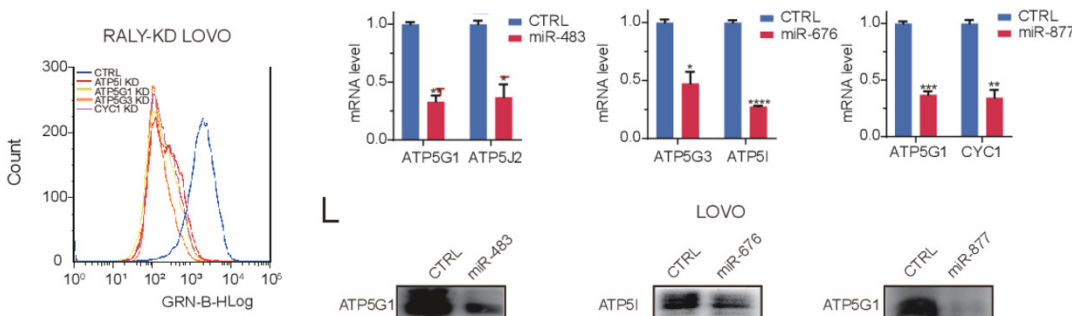

M
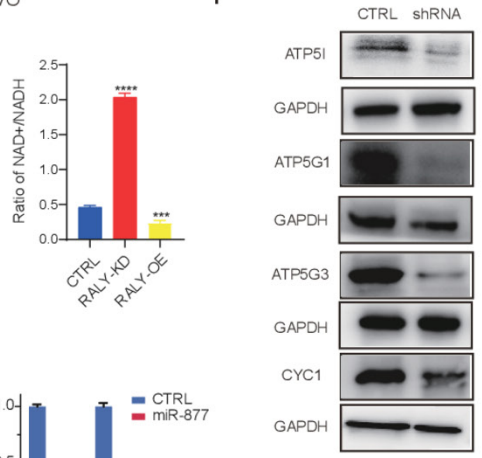

M RALY-KD LOVO
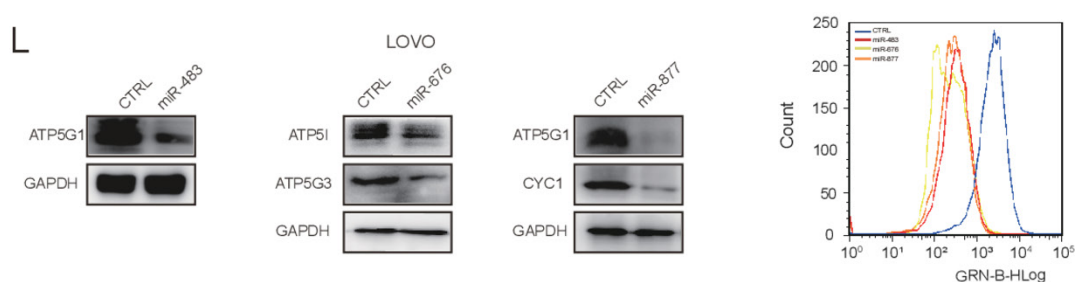

Figure 3 RALY reprogammes mitochondrial metabolism in CRC through mediating miRNA expression. (A) KEGG enrichment analysis of genes altered by RALY in HCT116 cells. (B, C) Depletion of RALY promoted expression of mitochondrial metabolism-related genes, including ATP5I, ATP5G1, ATP5G3 and CYC1 in mRNA level (B) and protein level (C). (D) Various levels of RALY affected ECAR value and glycolytic capacity in LOVO cells. (E) Various levels of RALY affected OCR value and mitochondrial respiratory capacity in LOVO cells. (F-H) Various levels of RALY regulated ATP production (F), ROS production (G) and NAD+/NADH level (H) in LOVO cells. (I) Knockdown of ATP5I, ATP5G1, ATP5G3, CYC1 in LOVO cell lines by lentiviral shRNA sequences (shATP5I, shATP5G1, shATP5G3, shCYC1), respectively. The knockdown effect was verified by protein level. (J) Knockdown of RALY-dependent ATP5I, ATP5G1, ATP5G3 and CYC1 decreased ROS levels in RALY-depleting LOVO cells. (K-L) Validation of RALY-dependent miRNAs in targeting mitochondrial metabolism-related genes at mRNA level (K) and protein level (L) in LOVO cells. (M) Overexpression of RALYdependent miRNAs decreased ROS levels in RALY-depleting LOVO cells. Data information: in all relevant panels, ${ }^{*} p<0.05 ;{ }^{* *} p<0.01 ;{ }^{* * *} p<0.001$; $* * * * p<0.0001$; two-tailed t-test. Data are presented as mean $\pm S D$ and are representative of three independent experiments. ATP, adenosine triphosphate; CRC, colorectal cancer; ECAR, extracellular acidification rate;. 
A

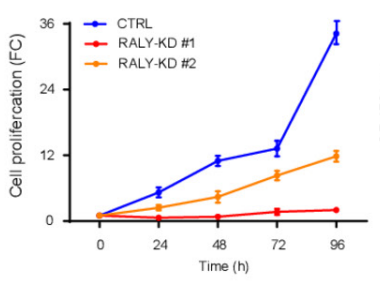

B

E

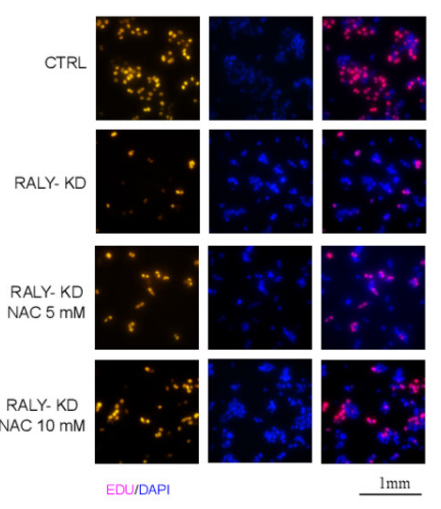

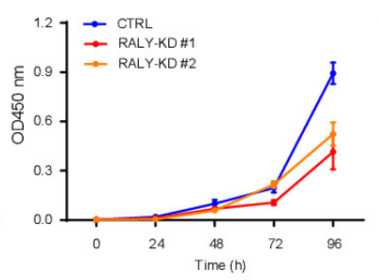

E-1

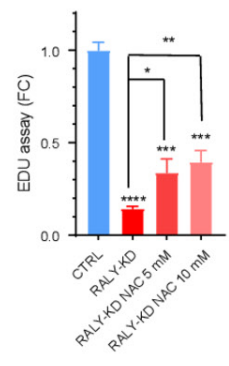

C

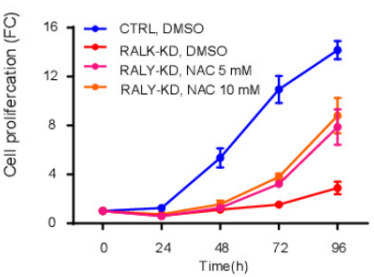

F

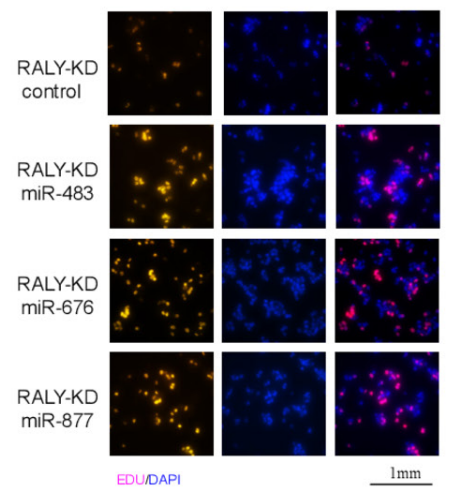

F-1

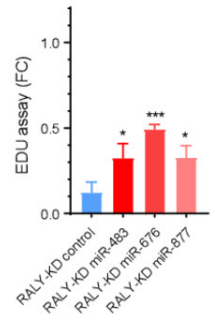

$\mathrm{H}$

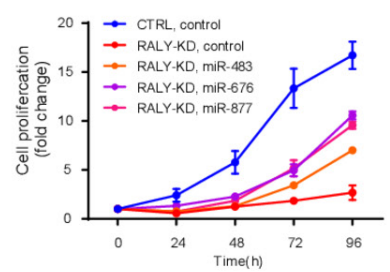

G
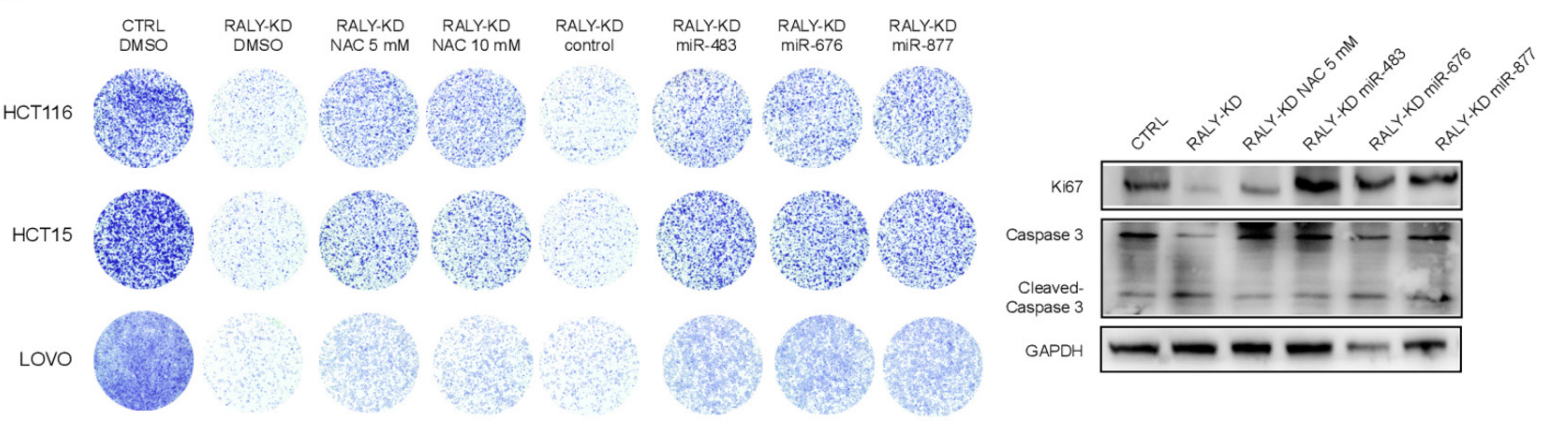

I

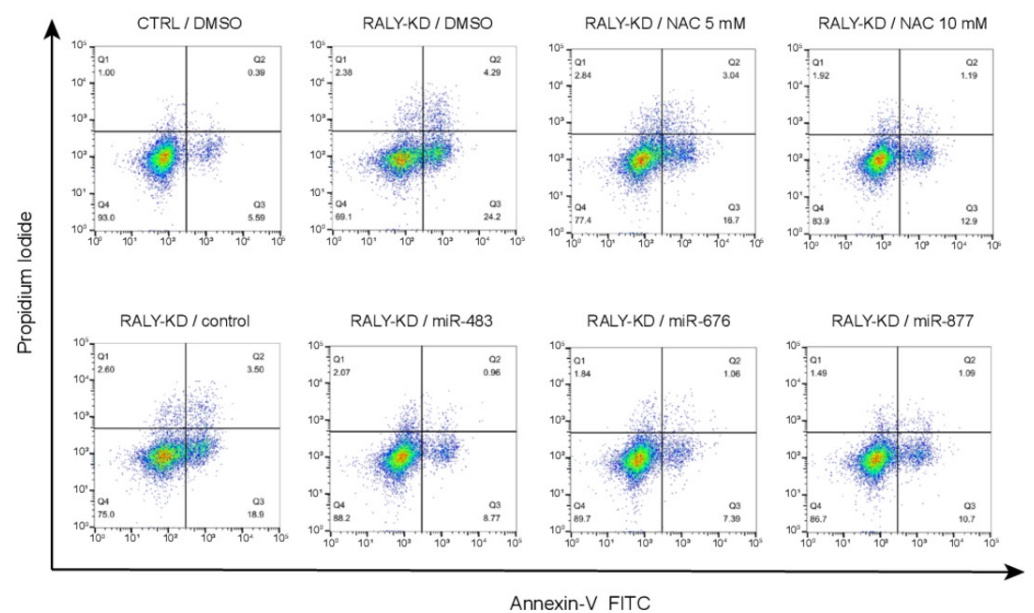

J

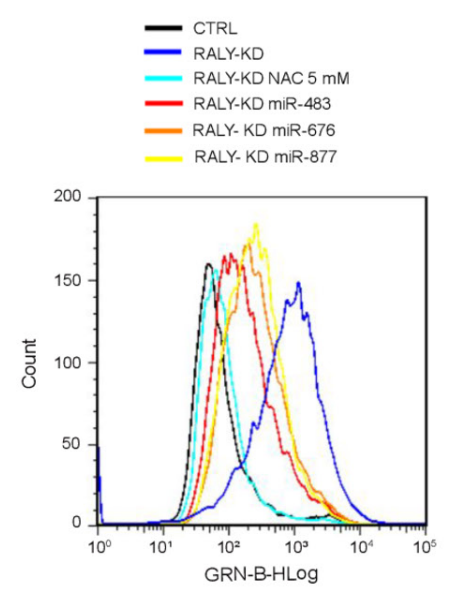

Figure 4 RALY regulates CRC cell proliferation and apoptosis under ROS stress. (A, B) Cell proliferation with RALY knockdown was measured by cell number counting (A) and CCK8 assay (B). (C) N-acetyl L-cysteine (NAC) treatments rescued cell proliferation in RALY-knockdown HCT116 cells. NAC is a scavenger of ROS. (D) Overexpression of ETC genes-related miRNAs rescued cell proliferation in RALY-knockdown HCT116 cells. (E, F) Treatment with NAC (E) or overexpression with miRNAs (F) rescued cell proliferation in RALY-knockdown HCT116 cells by EDU assay. Quantifications of fold change are shown in E-1 and F-1. (G) Cell survival assay of RALY-knockdown CRC cells after treated with NAC or overexpressed RALY-dependent miRNAs by crystal violet staining assay. (H) Knockdown of RALY promoted the cleavage of caspase 3 and increased Ki67 expression in HCT116 cells. (I) Knockdown of RALY significantly increased cell apoptosis in HCT116 cells by FACS. (J) Treatment with NAC or overexpression with miRNAs decreased ROS levels in RALY-knockdown HCT116 cells. Data information: in all relevant panels, ${ }^{*} p<0.05$; ${ }^{* *} p<0.01$; ${ }^{* * *} p<0.001$; ${ }^{* * *} p<0.0001$; two-tailed t-test. Data are presented as mean \pm SD and are representative of three independent experiments. CRC, colorectal cancer; ETC, electron transport chain. 
A

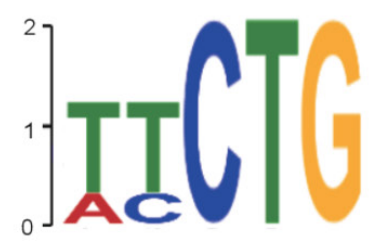

D

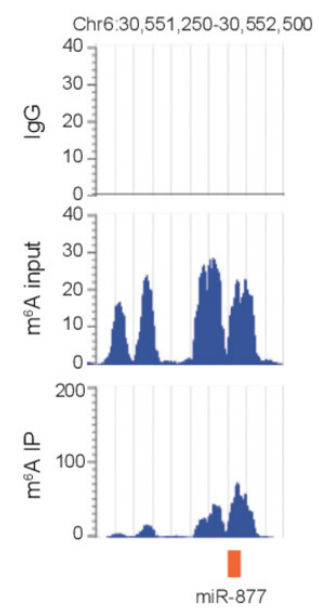

B

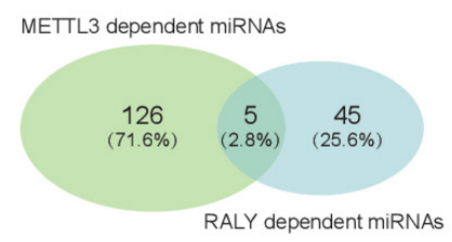

$E$

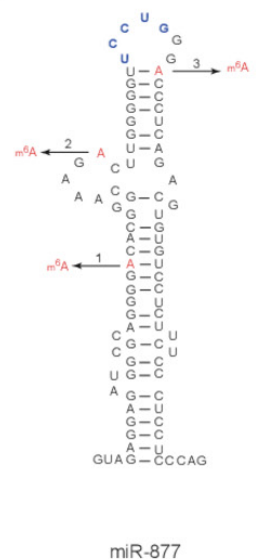

C
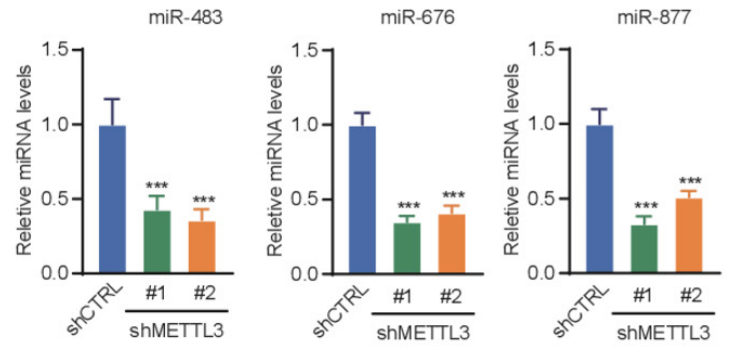

$\mathrm{F}$

G

Figure 5 RALY mediates miRNA processing through N6-methyladenosine switch. (A) Enrichment of HHCTG motif from the RALY HITS-CLIP peaks $(p=2.34 E-7)$. (B) Venn diagram depicting the intersection of miRNAs that are overlapped by RALY-knockdown and METTL3-knockdown (GSE70061). (C) Knockdown of METTL3 decreased RALY-dependent miRNAs in HCT116 cells. (D) $\mathrm{m}^{6} \mathrm{~A}$-IP sequencing proves that $\mathrm{m}^{6}$ a modification participates in regulation of RALY-dependent miR-877. (E) Three putative $m^{6}$ a sites and the HHCTG motif in miR-877. (F) RNA pulled down with $m^{6}$ a methylated oligonucleotides. (G) Illustration of the $\mathrm{m}^{6} \mathrm{~A}$-switch model mediated by RALY. Data information: in all relevant panels, ${ }^{* *} \mathrm{p}<0.01 ;{ }^{* * *} \mathrm{p}<0.001$; twotailed t-test. Data are presented as mean \pm SD and are representative of three independent experiments.

controlling CRC cell growth and cell death by mediating mitochondrial metabolism.

\section{RALY mediates miRNA processing through N6- methyladenosine switch}

We next looked into the detailed molecular mechanism by which RALY efficiently recruits pri-miRNAs to the Drosha complex and promotes their processing. It has been previously known that the N6-methyladenosine $\left(\mathrm{m}^{6} \mathrm{~A}\right)$ mark is associated with the processing of nuclear transcripts, ${ }^{31}$ and alternative splicing. ${ }^{32}$ The $\mathrm{m}^{6} \mathrm{~A}$ modification is able to alter RNA structure to enhance HNRNPC binding, ${ }^{33}$ and HNRNPA2B1 could bind to $\mathrm{m}^{6} \mathrm{~A}$ marks in some pri-miRNA transcripts to promote their processing. ${ }^{34}$ As a member of the HNRNPC subfamily, RALY shares an RRM motif and an acidic-rich auxiliary domain for formation of a strong and specific RNA interaction. ${ }^{35}$ We postulated that RALY may promote pri-miRNA processing via the $\mathrm{m}^{6} \mathrm{~A}$ modification of the pri-miRNA. We identified the binding motif of RALY (HHCTG) in the pri-miRNAs from the RALY-RIP sequencing (figure 5A). By comparing the miRNA profiling in the METTL3-depleted cells (GSE70061) and RALY-depleted cells, 5 miRNAs were found in the overlapped regions, including miR-877 (figure 5B and online supplemental figure 7A). Knockdown of METTL3 downregulated the expression levels of miR-483, miR-676 and miR-877 in CRC cells (figure 5C and online supplemental figure $7 \mathrm{~B})$. The $\mathrm{m}^{6} \mathrm{~A}$ signal is enriched in pri-miR-877, supporting the potential role of $\mathrm{m}^{6} \mathrm{~A}$ modification in the pri-miRNA processing (figure 5D). As the RGAC motif is the recognition motif for METTL3-mediated $\mathrm{m}^{6} \mathrm{~A}$ methylation, ${ }^{34}$ three potential $\mathrm{m}^{6} \mathrm{~A}$ sites on miR-877 were identified and indicated as A1, A2 and A3 (figure 5E). We performed RNA pull-down assay with methylated oligonucleotides to validate the authentic $\mathrm{m}^{6} \mathrm{~A}$ sites. The results showed that only $\mathrm{m}^{6} \mathrm{~A}$-methylation on $\mathrm{A} 3$ could significantly increase the pull-down amount of RALY (figure $5 \mathrm{~F}$ and online supplemental figure $7 \mathrm{C}$ ), and $\mathrm{A} 3$ locates at the terminal loop region of miR-877. Collectively, these results validate that $\mathrm{m}^{6} \mathrm{~A}$ modification could alter its local RNA structure at the terminal loop region of pri-miRNA and enhance the accessibility of its nearby region to modulate RALY binding, thus promoting processing of miRNAs (figure $5 \mathrm{G}$ ).

\section{METTL3 regulates RALY-mediated mitochondrial metabolism in CRC}

Since METTL3-mediated $\mathrm{m}^{6} \mathrm{~A}$ modification is involved in the RALY-dependent miRNA processing, we next asked whether METTL3 plays a role in the RALY-mediated mitochondrial metabolism. By comparing METTL3-dependent $\mathrm{m}^{6} \mathrm{~A}$ gene peaks (GSE94613) and RALY-dependent gene peaks, 454 altered genes were identified in the overlapped region. The GSEA analysis highlighted the overlapped genes enriched in metabolismrelated pathways, including OXPHOS (figure 6A). Depletion of METTL3 by siRNAs remarkably reduced the expression of miR-483, miR-676 and miR-877 in the RALY-overexpressed HCT116 cells (figure 6B). We next performed RIP assays to detect whether the pri-miRNA-binding activity of RALY is affected by METTL3. Significantly decreased levels of pri-miR483, pri-miR-676 and pri-miR-877 were bound by RALY after METTL3 depletion in HCT116 cells (figure 6C). We further 
A

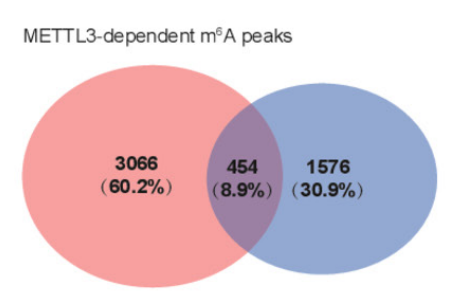

RALY-dependent gene peaks
RALY-dependent gene peaks

Description

(134) Metabolism of RNA

(110) mitochondrion organization (101) RNA splicing

(103) cellular component disassembly

(36) translational termination

(37) Respiratory electron transport, ATP synthesis

(38) oxidative phosphorylation

(105) regulation of mitotic cell cycle

(88) DNA repair

(70) regulation of cell growth

(91) apoptotic signaling pathway

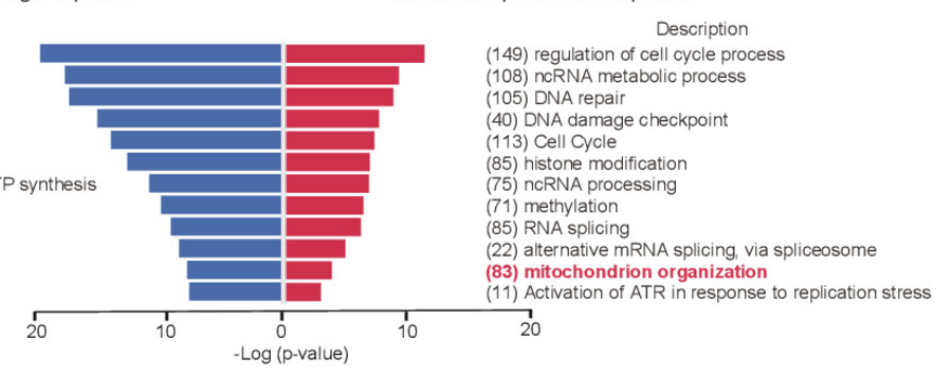

C

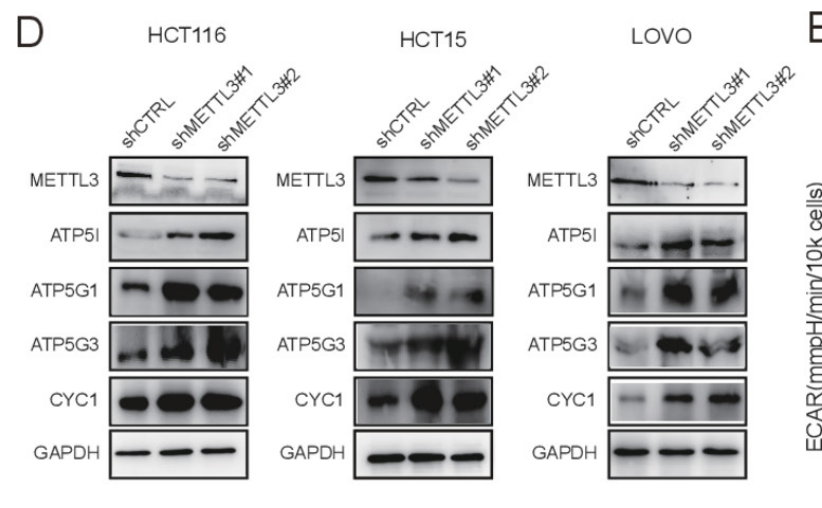

F

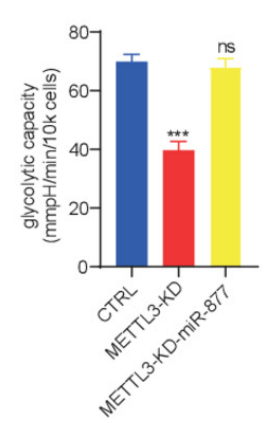

HCT116

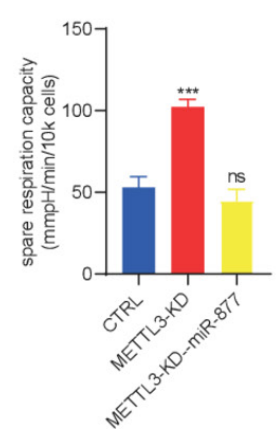

G

HCT116

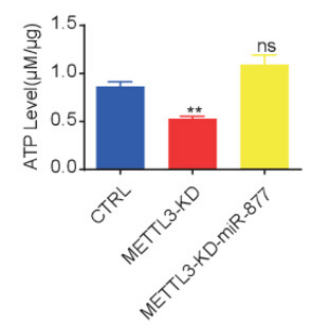

miR-877

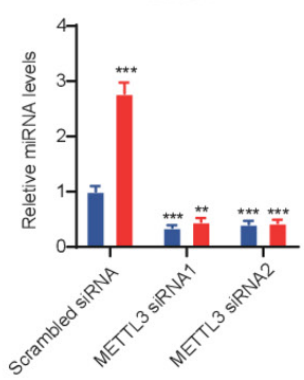

- $\lg \mathrm{G}$

- RALY (Scrambled siRnA)

- raly (MEttl3 sirna1)

- CTRL

- Raly-oe

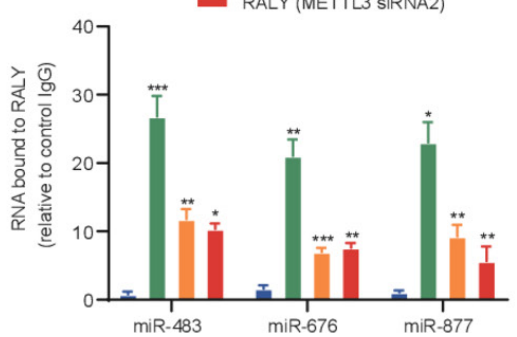

E
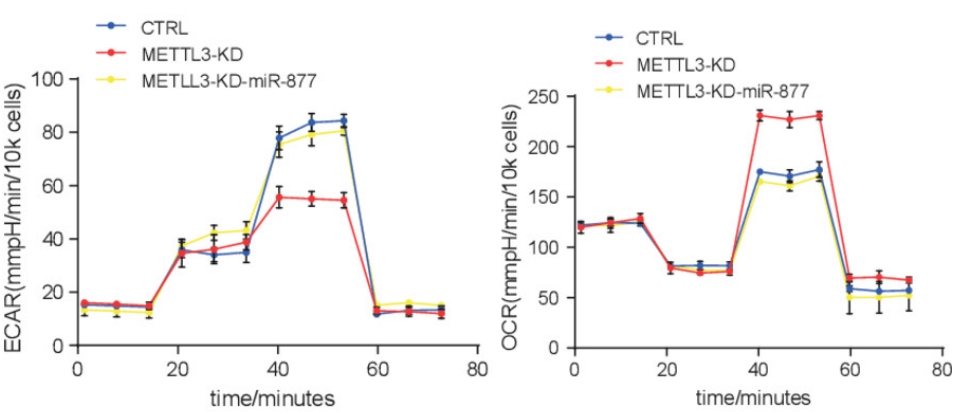

$H$

HCT116

|

HCT116
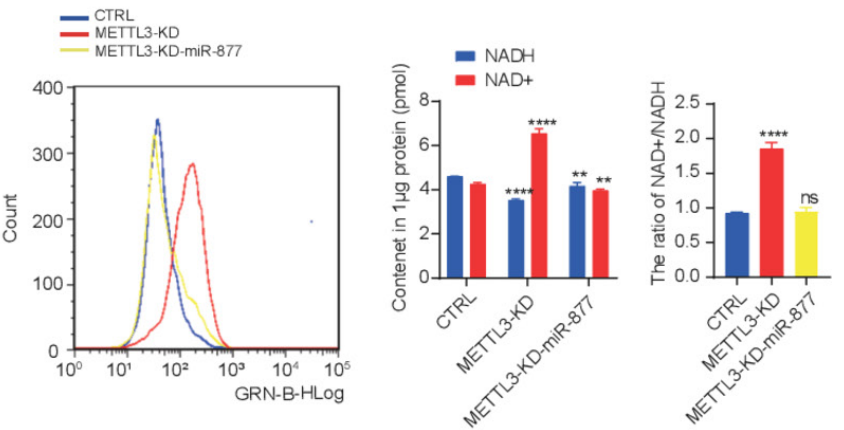

Figure 6 METTL3 regulates RALY-mediated mitochondrial metabolism in CRC. (A) The mitochondrial metabolic pathway is highlighted in both RALYdependent genes and METTL3-dependent genes. (B) Knockdown of METTL3 by siRNAs abolished the induction of RALY-dependent miRNAs expression in RALY-overexpressed HCT116 cells. (C) Knockdown of METTL3 by siRNAs diminished the interaction of RALY with pri-miRNAs. RIP assays were performed using anti-RALY antibodies and the lysates of HCT116 cells treated with or without METTL3 siRNAs. (D) Knockdown of METTL3 increased the expression of RALY-dependent ATP5I, ATP5G1, ATP5G3 and CYC1 by Western blotting. (E) Overexpression of miR-877 rescued ECAR value and OCR level in METTL3-knockdown HCT116 cells. (F) Overexpression of miR-877 rescued glycolytic capacity and mitochondrial respiratory capacity in METTL3-knockdown HCT116 cells. (G-I) Overexpression of miR-877 rescued ATP production (G), ROS production (H) and NAD+/NADH level (I) in METTL3-knockdown HCT116 cells. Data information: in all relevant panels, ${ }^{*} p<0.05$; ${ }^{* *} p<0.01 ;{ }^{* *} p<0.001$; ${ }^{* * * *} p<0.0001$; two-tailed t-test. Data are presented as mean \pm SD and are representative of three independent experiments. 
generated stable METTL3-knockdown models in HCT116, HCT15 and LOVO cells with two independent shRNA sequences (shMETTL3\#1 and \#2). Knockdown of METTL3 significantly suppressed cell growth in CRC cells (online supplemental figure $8 \mathrm{~B})$. Accordingly, depletion of METTL3 induced the expression of ATP5I, ATP5G1, ATP5G3 and CYC1 at both protein and RNA levels (figure 6D and online supplemental figure 8A). Loss of METTL3 promoted mitochondrial respiration as indicated by increased OCR levels, and suppressed aerobic glycolysis as indicated by decreased ECAR levels in CRC cells. Overexpression of miR-877 rescued the effects on mitochondrial respiration and aerobic glycolysis caused by METTL3 depletion (figure 6E,F and online supplemental figure 8C,D). In line with it, knockdown of METTL3 significantly reduced ATP production and enhanced ROS levels, while overexpression of miR-877 diminished the effects caused by the METTL3 knockdown (figure 6G,H and online supplemental figure $8 \mathrm{EF}$ ). A notable increase in the $\mathrm{NAD}+/ \mathrm{NADH}$ ratio was observed in the METTL3-depleted CRC cells, but overexpression of miR-877 abolished the induction (figure 6I and online supplemental figure 8G). These results suggest that METTL3-dependent $\mathrm{m}^{6} \mathrm{~A}$ modification in pri-miRNAs is essential for the RALY-mediated mitochondrial metabolism in CRC cells.

\section{RALY serves as a potential therapeutic target in CRC}

We next examined the functional role of RALY in tumourigenesis in vivo. We generated inducible RALY-knockdown syngeneic mouse tumour models by subcutaneously injection of mouse MC38 cells into C57BL/6 mice (figure 7A). After initial tumour establishment, administration of doxycycline $(1.0 \mathrm{mg} /$ $\mathrm{mL}$ ) in drinking water suppressed RALY expression and consequently inhibited tumour growth (figure 7B-E). Knockdown of RALY in the tumours induced the expression of ATP5I and decreased miR-676 expression, as indicated by Western blotting assay and RT-PCR, respectively (figure 7B,C). We then established an orthotopic mouse xenograft CRC model by injection of inducible RALY-knockdown LOVO cells into the cecal wall of NOD-SCID mice. The group treated with doxycycline showed fewer and smaller tumour nodules in the colon compared with the group treated with water (figure 7F and online supplemental figure $9 \mathrm{~A}$ ), as indicated by the H\&E staining of the colon and tumour weight (figure $7 \mathrm{G}$ and $\mathrm{H}$ and online supplemental figure 9B). Using the organoid models generated from the MC38derived tumours, we find that knockdown of RALY by treatment with doxycycline could significantly inhibit the growth of the organoids (figure 7I,J), and induced apoptosis in the organoid (figure $7 \mathrm{~K}$ ). To verify whether the inhibitory effect by RALY in vivo is mediated by RALY-dependent miRNAs, we overexpressed miR-676 in the RALY-knockdown HCT116 cells. Notably, overexpression of miR-676 in the RALY-knockdown HCT116 cells partly rescued xenograft tumour growth (figure $7 \mathrm{~L}-\mathrm{N}$ ), and suppressed the expression of ATP5I in comparison with the RALY-knockdown xenograft tumours, as indicated by immunohistochemistry (IHC) and Western blotting assays (figure 7O-Q and online supplemental figure 9C).

Further, we generated CRC patient-derived xenograft (PDX) models and depleted RALY expression by using RNAi and nano-gold particle delivery tools (figure 8A). We employed two treatment strategies for the PDX models. The first was to administrate with the RNAi regimen on the second day after the tumours were embedded, while the second was to administrate with the RNAi regimen on the 20th day when the tumours regained growth to the size of $100 \mathrm{~mm}^{3}$. Our results show that both treatment strategies led to significantly reduced tumour growth (figure 8B-D) and induced the expression of ATP5I, as indicated by the $\mathrm{H} \& \mathrm{E}$ and $\mathrm{IHC}$ staining assays (figure $8 \mathrm{E}, \mathrm{F}$ ). We also established the patient-derived organoid (PDO) models from fresh CRC patient tumours. Treatment with siRALY dramatically reduced the organoid viability and made it vulnerable in shape and membrane permeability (figure $8 \mathrm{G}, \mathrm{H}$ ), and induced apoptosis phenotype in the model (figure 8I). To determine whether various levels of RALY would affect the therapeutic effects of siRNA treatments, we select 3 CRC patient tissues with various levels of RALY (online supplemental figure 10A). Targeting RALY by siRNAs in CRC tissue with high level of RALY achieved the maximal inhibitory effects on tumour growth in both PDO model and PDX model, while it also showed effective inhibition of tumour growth in CRC tissues with normal or low level of RALY (online supplemental figure 10B-E).

In analysis of clinical database (TCGA-COAD), the expression levels of RALY and METTL3 are significantly higher in tumours compared with normal tissues, while the levels of ATP5I, ATP5G1 and ATP5G3 are significantly lower in tumours (online supplemental figure 11A). Lower expression levels of these ETC genes indicate poorer clinical outcome for CRC patients (figure 8J). Further, we showed the positive correlation between RALY and its dependent miRNAs in CRC clinical samples from various CRC datasets (online supplemental figure 11BC) and (online supplemental table 4). Higher expression level of miR-877 and miR-483 shows significant poorer clinical outcome for CRC patients, which is consistent with higher expression level of RALY (online supplemental figure 11DE). Collectively, these results suggest that RALY promotes tumour growth in vivo and in the organoid models, highlighting its role as a potential therapeutic target in CRC.

\section{DISCUSSION}

Systematic analysis of genome-wide miRNA expression profiles indicates that aberrant miRNA expression contributes to cellular transformation and tumour progression. ${ }^{36}{ }^{37}$ The stem-loop structure of miRNA was found to be critical for the Droshamediated cleavage. ${ }^{38} 39$ Current studies, including ours, have shown that RNA-binding proteins confer the specificity for recruiting and processing individual miRNAs. ${ }^{34} 40-43$ A growing list of RNA-binding proteins identified from the Drosha microprocessor complex is the members of the heterogeneous nuclear ribonucleoprotein family, including hnRNPA1, hnRNPA2B1, hnRNPK and hnRNPL. ${ }^{34}{ }^{43-45}$ In this study, we identify a novel member of hnRNP, RALY, which is highly associated with CRC stages and aggressiveness, interacts with the Drosha complex and promotes the expression of a subset of miRNAs including miR-483, miR-676 and miR-877. Previously miR-483 was reported to be involved in initiation and invasion of several cancers. $^{46-48}$ We show that these RALY-dependent miRNAs downregulate the ETC genes, ATP5I, ATP5G1, ATP5G3 and CYC1, and mediate reprogramming of mitochondrial metabolism in CRC cells, which provides the functional link between epigenetic regulation and mitochondrial metabolism. Our results demonstrate that inhibition of RALY abolishes ATP production, induces ROS level and increases the NAD+/NADH ratio in CRC cells, while overexpression of miR-483, miR-676 and miR-877 in the RALY-depleted cells could rescue the metabolic phenotype. We further knocked down the related ETC genes in RALYdepleted cells and validated that the metabolism-centric effects of RALY in OXPHOS are mainly mediated by these ETC genes (figure 9). 
A

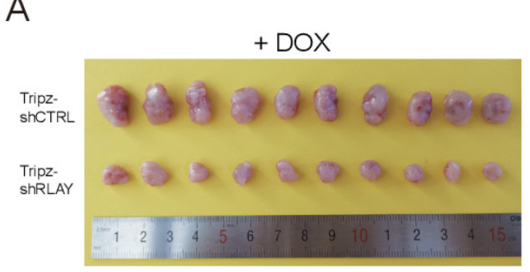

B
E

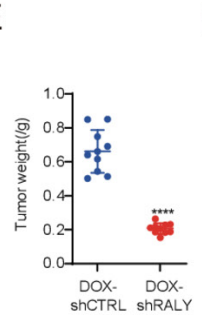

F

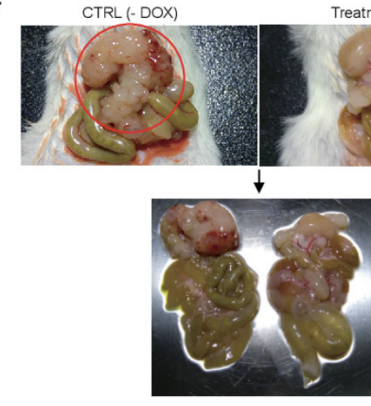

I
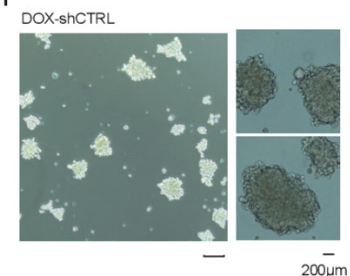

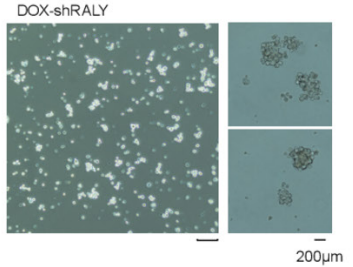

L
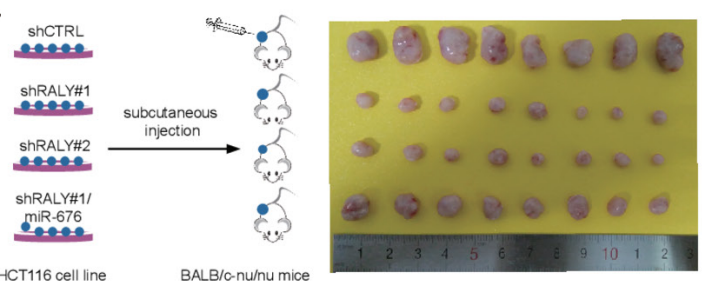

M

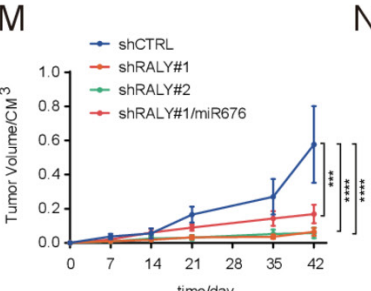

N

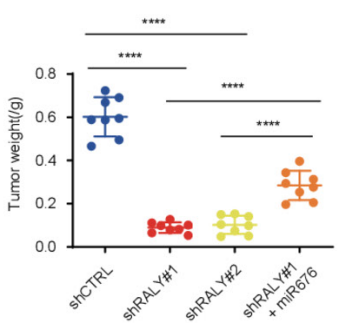

0
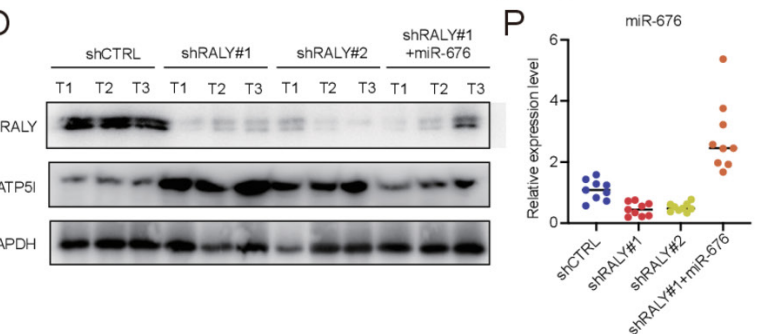

C

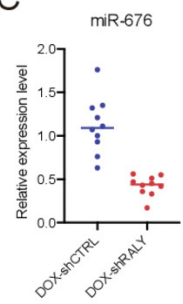

D
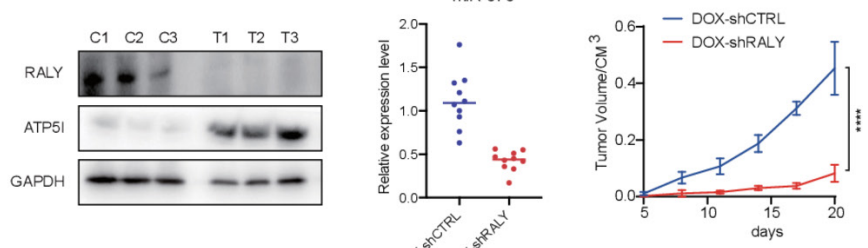

$\mathrm{H}$
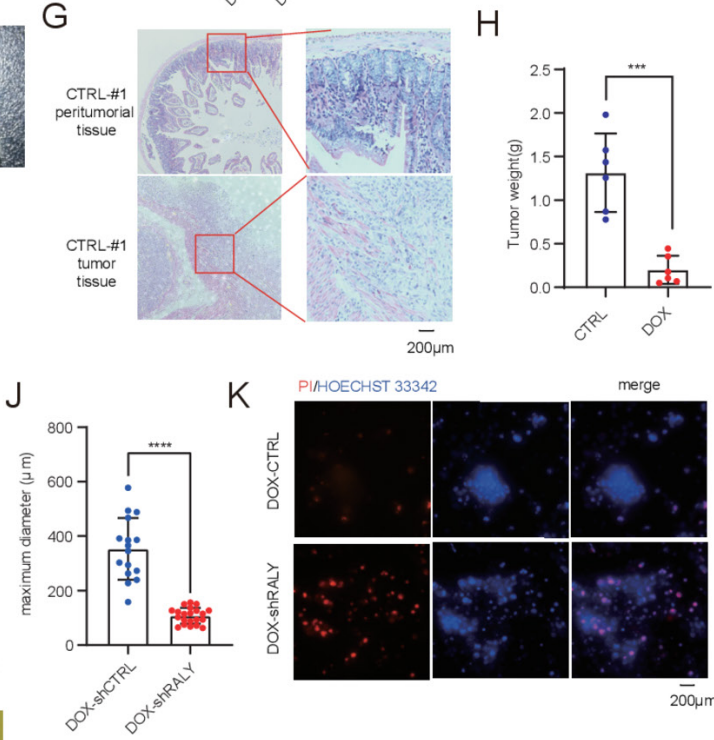

Q
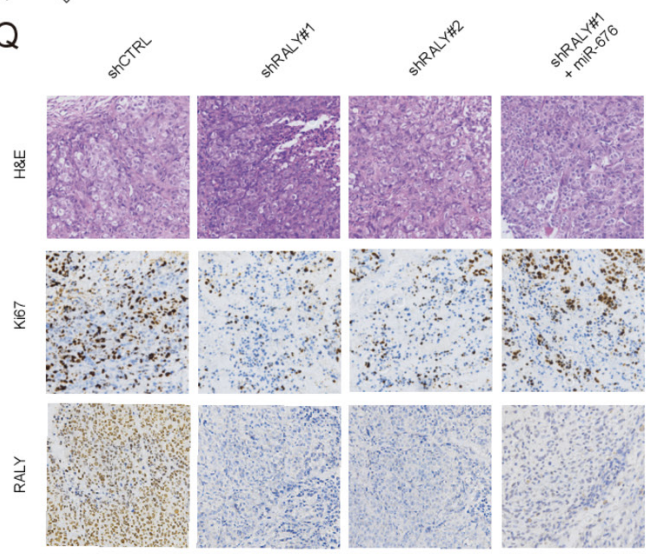

\section{要}
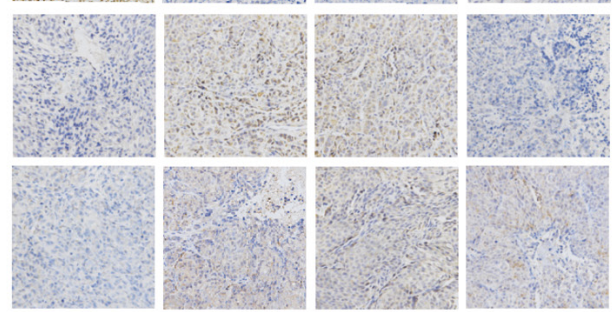

200 um

Figure 7 Depletion of RALY inhibits tumour growth in vivo and in the CRC organoid model. (A) Depletion of RALY by Dox administration suppressed tumour growth in MC38 cells derived xenografts. (B) Validation of RALY and ATP5I expression level in inducible knockdown of RALY in MC38 cells derived xenografts by Western blotting. (C) Downregulation of miR-676 was validated in RALY-knockdown MC38 xenografts. (D, E) Tumour growth curve (D) and tumour weight (E) of MC38 cells derived xenografts treated with Dox. (F-H) Tumour images of orthotopic xenograft mice model (F) and H\&E staining of LOVO tumour and pericarcinomatous tissue (G). Tumour weight of orthotopic xenograft is shown (H). (I, J) DOX-induced knockdown of RALY suppressed growth of MC38-derived tumour organoids (I). Quantification of size of organoids is shown (J). (K) DOX-induced knockdown of RALY induced apoptosis of MC38-derived tumour organoids. (L) Overexpression of miR-676 rescued tumour growth in RALY-knockdown HCT116 cells derived xenografts. $(M, N)$ Tumour growth curve $(M)$ and tumour weight $(N)$ of RALY-knockdown HCT116 cells derived xenografts treated with overexpression of miR-676. (0-Q) Validation of RALY, ATP5I and miR-676 expression level in knockdown of RALY by overexpression of miR676 in HCT116 cells derived xenografts by Western blotting (0), RT-PCR (P) and IHC (Q). Data information: in all relevant panels, *** $p<0.001$; $* * * * p<0.0001$; two-tailed t-test. Data are presented as mean \pm SD. CRC, colorectal cancer; IHC, immunohistochemistry. 
A

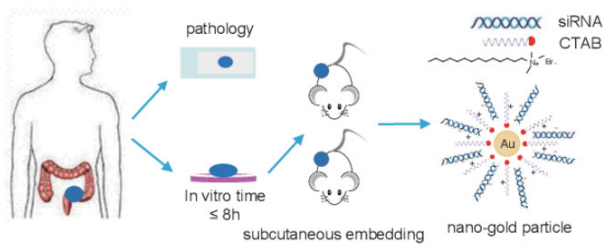

B

CRC-PDX\#1

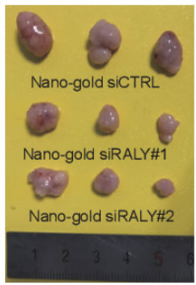

C

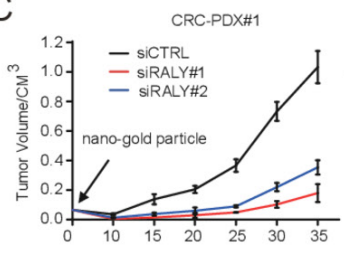

D

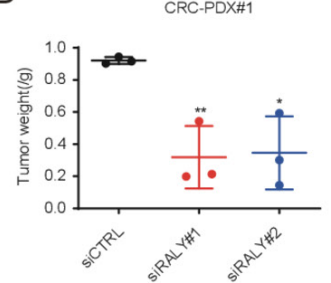

$\mathrm{H}$
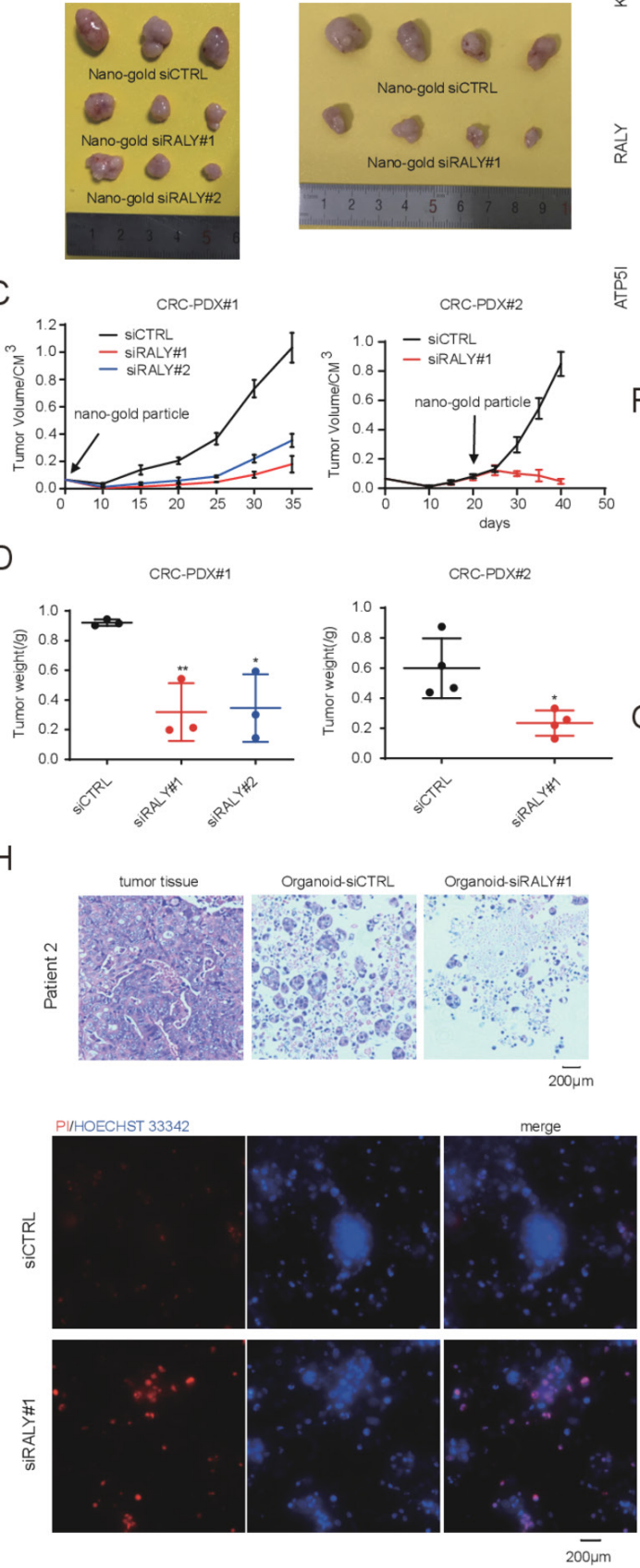

CRC-PDX\#2

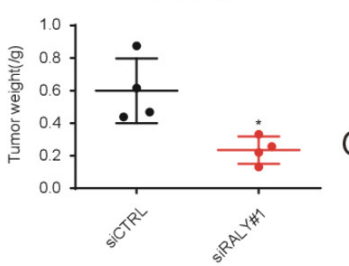

E
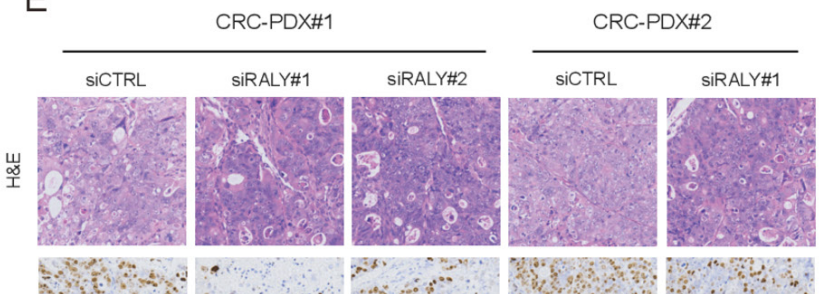

产:
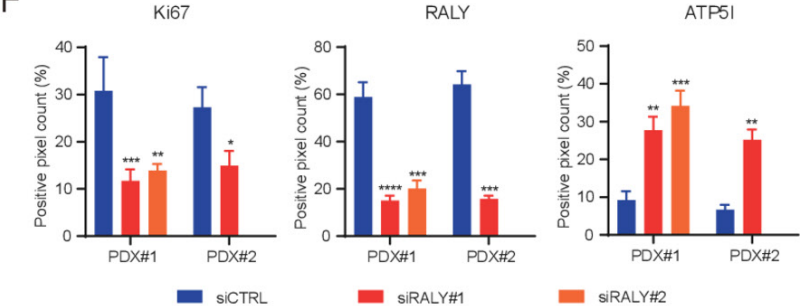

G
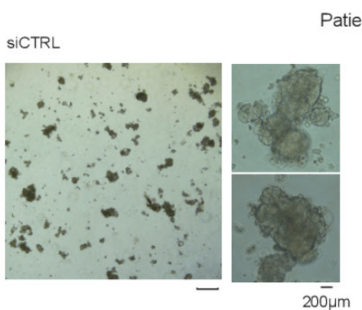

atient 2

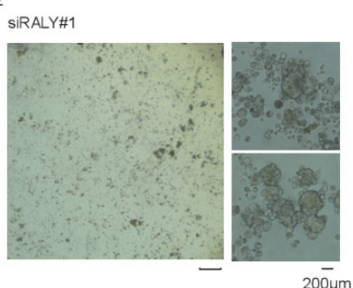

J
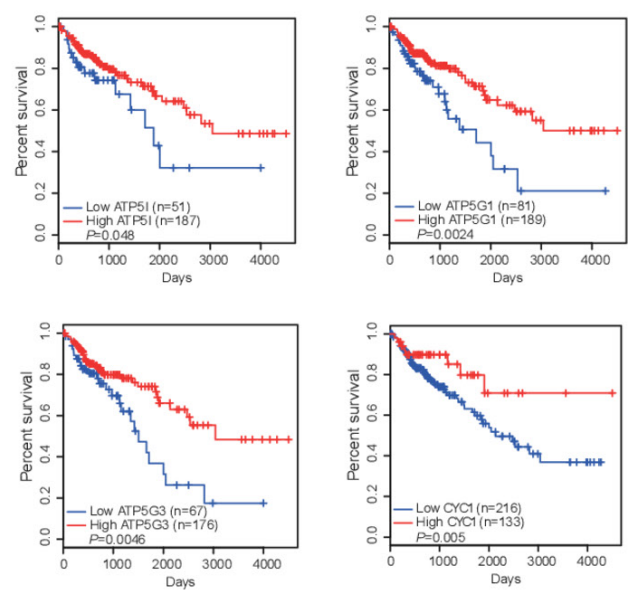

Figure 8 RRALY serves as a potential therapeutic target in CRC. (A) Schematic diagram of human CRC PDX mouse model based RALY siRNA treatment regimen. CTAB-nano-gold siRNA particles are generated as indicated. (B-D) Gross tumour images (B), tumour growth curve (C), and tumour weight (D) of PDX model treated with nano-gold siCTRL and nano-gold siRALY with two administration strategies as indicated. The nano-gold particles $(20 \mathrm{mg} / \mathrm{kg}$ ) were administrated every 3 days for 3 weeks. (E, F) H\&E and IHC images of PDX tumours (E) and quantification of RALY and ATP5I expression was analysed in IHC images by Image Pro Plus (IPP) analysis (F). (G) Knockdown of RALY by siRNA suppressed growth of CRC patientderived tumour organoids. (H) H\&E images of CRC patient-derived tumour organoids treated with RALY siRNAs. (I) Knockdown of RALY by siRNA induced apoptosis of CRC patient-derived tumour organoids. (J) Down-regulation of ATP5I, ATP5G1, ATP5G3 and CYC1 are significantly associated with shorter overall survival rates in CRC patients. Data information: in all relevant panels, ${ }^{*} \mathrm{p}<0.05$; ${ }^{* *} \mathrm{p}<0.01$; two-tailed t-test. Data are presented as mean \pm SD. CRC, colorectal cancer PDX, patient-derived xenograft. 


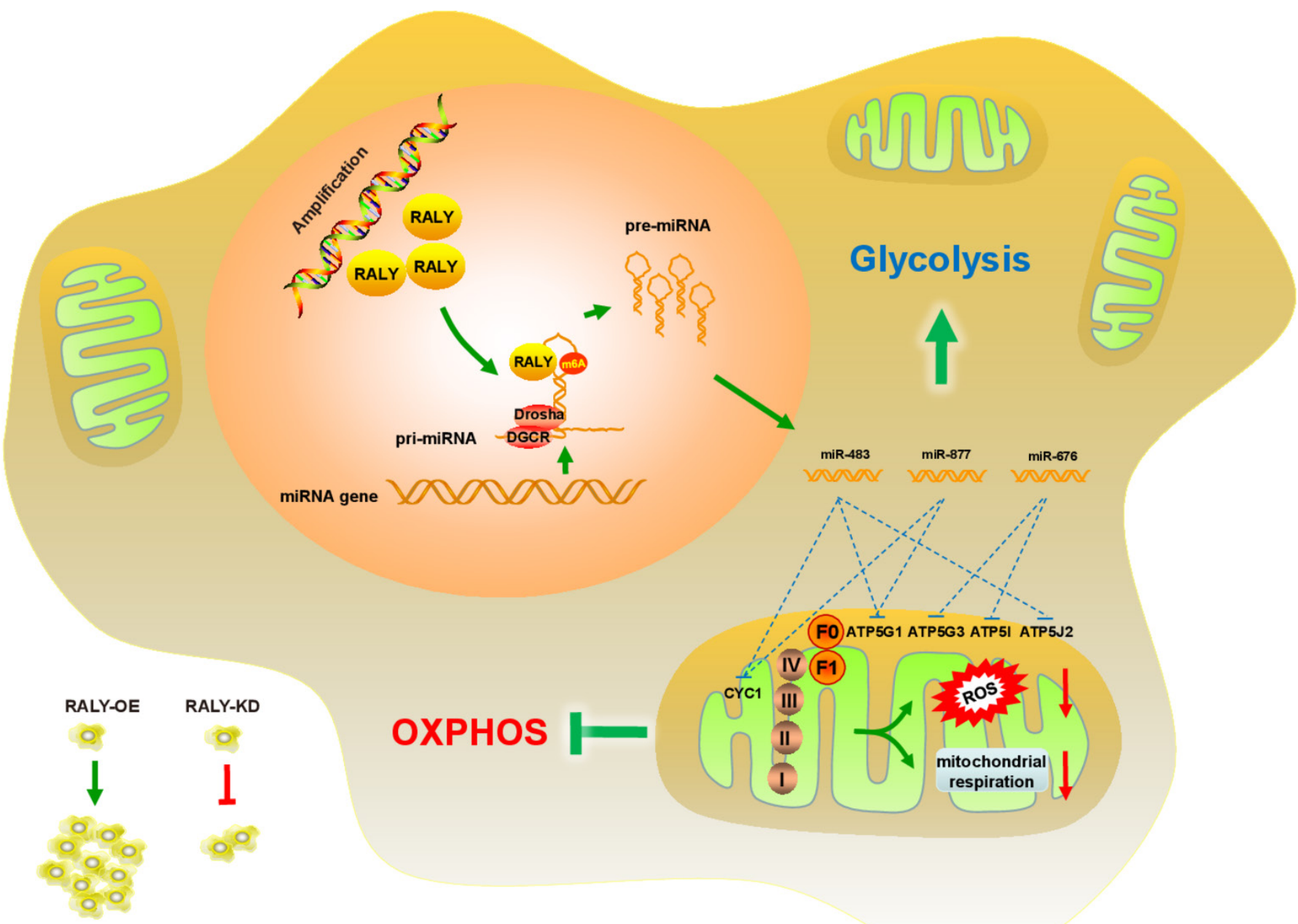

Figure 9 Working model. RALY is amplified in most CRC cells. Overexpressed RALY recruits to Drosha/DGCR8 complexes and promotes a subset of miRNAs processing by the $\mathrm{m}^{6}$ a switch. The upregulated miRNAs inhibit oxidative phosphorylation (OXPHOS) by targeting key players in the mitochondrial respiration, leading to cell proliferation in CRC with RALY overexpression. CRC, colorectal cancer.

Recently, N6-methyladenosine modification has been shown as critical post-transcriptional regulation for gene expression in tumour development, and plays important roles in RNA splicing and degradation. ${ }^{49-52}$ Previous studies reported that hnRNP proteins promoted some miRNAs processing by recognising the $\mathrm{m}^{6} \mathrm{~A}$-modified pri-miRNAs transcripts. ${ }^{32-34} \mathrm{As}$ a member of the hnRNPC subfamily, RALY is now found to recognise the terminal loop region of pri-miR- 877 with $\mathrm{m}^{6} \mathrm{~A}$ modification, and facilitate the binding of pri-miR- 877 to the microprocessor complex, thus promoting its processing. Knockdown of METTL3, a primary $\mathrm{m}^{6} \mathrm{~A}$ methylase, abolishes the upregulation of RALY-dependent miRNAs, and in part affects the RALY-mediated mitochondrial metabolism in CRC cells. Whether RALY acts as a direct $\mathrm{m}^{6} \mathrm{~A}$ reader or modulates the miRNA processing through the $\mathrm{m}^{6} \mathrm{~A}$ switch raised an intriguing question. Previously, hnRNPA2B1 was reported as a direct $\mathrm{m}^{6} \mathrm{~A}$ reader to promote some miRNAs processing. ${ }^{34}$ However, subsequent structure analysis suggested that hnRNPA2B1 may promote pri-miRNAs processing through the $\mathrm{m}^{6} \mathrm{~A}$ switch instead of acting as a direct $\mathrm{m}^{6} \mathrm{~A}$ reader. ${ }^{53}$ Of note, hnRNPC was reported to modulate alternative splicing and binding activities of target RNAs through the $\mathrm{m}^{6} \mathrm{~A}$-switchregulated mode to gain the abundance of gene expression. ${ }^{33}$ For RALY, we performed RNA pull-down assay with methylated oligonucleotides and found that the functional $\mathrm{m}^{6} \mathrm{~A}$ site locates at the terminal loop region of pri-miR-877, adjacent to the RALY-binding motif, indicating that RALY may mediate primiRNA processing through the $\mathrm{m}^{6} \mathrm{~A}$ switch mode.

Combinational therapy with BACH1 and ETC inhibitors showed favourable therapeutic effects in triple-negative breast cancer. ${ }^{11}$ Similarly, we demonstrate that RALY is required for the metastasis of aggressive CRC, and its gene expression is highly associated with poor clinical outcomes. Similar to BACH1, RALY functions as a negative regulator to regulate expression of the ETC genes and reprogramme mitochondrial metabolism, suggesting that it may be a promising target for cancer therapy. Inhibition of RALY sensitised resistant CRC cells to oxaliplatin. ${ }^{54}$ In terms of translational development, we developed a core-shell nano-gold platform for delivering in vivo optimised RALY siRNA to target RALY as our previous 'nano-bomb' platform. ${ }^{55}$ By using human PDX model and PDO model from clinical patient samples, we validated the significance of targeting the RALY-miRNAs-mediated mitochondrial metabolism axis with our nano-gold technology in CRC therapy. Taken together, our study not only reveals a crucial metabolism-centric role of RALY in reprogramming OXPHOS mediated by the $\mathrm{m}^{6} \mathrm{~A}$ switch, but also highlights a potential therapeutic target in CRC. 


\section{Author affiliations}

'National-Local Joint Engineering Laboratory of Druggability and New Drug Evaluation, National Engineering Research Center for New Drug and Druggability (cultivation), Guangdong Province Key Laboratory of New Drug Design and Evaluation, School of Pharmaceutical Sciences, Sun Yat-Sen University, Guangzhou, China

${ }^{2}$ Department of Gastrointestinal Surgery, The First Affiliated Hospital of Sun Yat-Sen University, Guangzhou, China

${ }^{3}$ Department of Medical and Molecular Genetics, Indiana University School of Medicine, Indianapolis, IN, USA

${ }^{4}$ Division of Pulmonary, Allergy and Critical Care Medicine, Department of Medicine, University of Pittsburgh, Pittsburgh, PA, USA

${ }^{5}$ School of Materials Science and Engineering, Sun Yat-Sen University, Guangzhou, China

${ }^{6}$ Center for Precision Medicine, Sun Yat-Sen University, Guangzhou, China ${ }^{7}$ Melvin and Bren Simon Cancer Center, Indiana University, Indianapolis, IN, USA ${ }^{8}$ School of Life Sciences, Beijing University of Chinese Medicine, Beijing, China ${ }^{9}$ State Key Laboratory of Biocontrol, Guangdong Province Key Laboratory of Pharmaceutical Functional Genes, School of Life Sciences, Sun Yat-Sen University, Guangzhou, China

\section{Acknowledgements The authors thank Dr Bo Wei for help with CRC tissue} isolation. They also acknowledge the support of the SYSU Animal Centre.

Contributors Study concept and design: GW, LS and AW; Acquisition of data: LS, AW, ZZ, DC, HL, CL, SY, YN, ZL and SZ; Analysis and interpretation of data: GW, AX $X L, L S$ and AW; Statistical analysis: LS, AW, ZZ and DC; Drafting of the manuscript: GW and LS; Critical revision and final approval of the manuscript: GW, XL and AX; Administrative, technical support: SW, XB and WH; Study supervision: GW, XL and AX.

Funding This work was supported in part by grants from the National Natural Science Foundation of China (82073869, 31701114, 21672266, 81871994, 81701834, 91942301); National Key Research and Development Project (2019YFC1710104) from the Ministry of Science and Technology of China; Guangdong Basic and Applied Basic Research Foundation (2019A050510019, 2019B151502063); Guangdong Provincial Key Laboratory of Construction Foundation (2017B030314030, 2020B1212060034); Guangzhou Science and Technology Planning Programme (202002020051, 201902020018); National Engineering Research Centre for New Drug and Druggability Evaluation, Seed Program of Guangdong Province (2017B090903004)

\section{Competing interests None declared.}

Patient consent for publication Not required.

Provenance and peer review Not commissioned; externally peer reviewed.

Data availability statement All data relevant to the study are included in the article or uploaded as online supplemental information. The authors are willing to share the data related to any of the work reported.

Supplemental material This content has been supplied by the author(s). It has not been vetted by BMJ Publishing Group Limited (BMJ) and may not have been peer-reviewed. Any opinions or recommendations discussed are solely those of the author(s) and are not endorsed by BMJ. BMJ disclaims all liability and responsibility arising from any reliance placed on the content. Where the content includes any translated material, BMJ does not warrant the accuracy and reliability of the translations (including but not limited to local regulations, clinical guidelines, terminology, drug names and drug dosages), and is not responsible for any error and/or omissions arising from translation and adaptation or otherwise.

Open access This is an open access article distributed in accordance with the Creative Commons Attribution Non Commercial (CC BY-NC 4.0) license, which permits others to distribute, remix, adapt, build upon this work non-commercially, and license their derivative works on different terms, provided the original work is properly cited, appropriate credit is given, any changes made indicated, and the use is non-commercial. See: http://creativecommons.org/licenses/by-nc/4.0/.

\section{ORCID iDs}

Xiongbin Lu http://orcid.org/0000-0002-7987-9825

Anlong Xu http://orcid.org/0000-0002-1419-3494

Guohui Wan http://orcid.org/0000-0001-5170-7282

\section{REFERENCES}

1 Hanahan D, Weinberg RA. Hallmarks of cancer: the next generation. Cell 2011;144:646-74.

2 Vander Heiden MG, Cantley LC, Thompson CB. Understanding the Warburg effect: the metabolic requirements of cell proliferation. Science 2009;324:1029-33.

3 Hattori A, Tsunoda M, Konuma T, et al. Cancer progression by reprogrammed BCAA metabolism in myeloid leukaemia. Nature 2017;545:500-4.
4 Levine AJ, Puzio-Kuter AM. The control of the metabolic switch in cancers by oncogenes and tumor suppressor genes. Science 2010;330:1340-4.

5 Han C, Yang L, Choi HH, et al. Amplification of USP13 drives ovarian cancer metabolism. Nat Commun 2016:7:13525.

6 Dawson MA, Kouzarides T. Cancer epigenetics: from mechanism to therapy. Cell 2012;150:12-27.

7 Kaelin WG, McKnight SL. Influence of metabolism on epigenetics and disease. Cell2013;153:56-69.

8 Lu C, Thompson CB. Metabolic regulation of epigenetics. Cell Metab 2012;16:9-17.

9 Vander Heiden MG, DeBerardinis RJ. Understanding the Intersections between metabolism and cancer biology. Cell 2017;168:657-69.

10 Weinberg SE, Chandel NS. Targeting mitochondria metabolism for cancer therapy. Nat Chem Biol 2015;11:9-15.

11 Lee J, Yesilkanal AE, Wynne JP, et al. Effective breast cancer combination therapy targeting Bach1 and mitochondrial metabolism. Nature 2019;568:254-8.

12 Birsoy K, Possemato R, Lorbeer FK, et al. Metabolic determinants of cancer cell sensitivity to glucose limitation and biguanides. Nature 2014;508:108-12.

13 Wheaton WW, Weinberg SE, Hamanaka RB, et al. Metformin inhibits mitochondrial complex I of cancer cells to reduce tumorigenesis. eLife2014;3:e02242.

14 Dawson MA. The cancer epigenome: concepts, challenges, and therapeutic opportunities. Science 2017;355:1147-52.

15 Taby R, Issa J-PJ. Cancer epigenetics. CA: A Cancer Journal for Clinicians 2010;60:376-92.

16 Hobert 0 . Gene regulation by transcription factors and microRNAs. Science 2008;319:1785-6.

17 Hatziapostolou M, Polytarchou C, Iliopoulos D. miRNAs link metabolic reprogramming to oncogenesis. Trends Endocrinol Metab 2013;24:361-73.

18 Gao P, Tchernyshyov I, Chang T-C, et al. C-Myc suppression of miR-23a/b enhances mitochondrial glutaminase expression and glutamine metabolism. Nature 2009:458:762-5.

19 Zhu H, Shyh-Chang N, Segrè AV, et al. The Lin28/let-7 axis regulates glucose metabolism. Cell 2011;147:81-94.

20 Trajkovski M, Hausser J, Soutschek J, et al. Micrornas 103 and 107 regulate insulin sensitivity. Nature 2011;474:649-53.

21 Jordan SD, Krüger M, Willmes DM, et al. Obesity-induced overexpression of miRNA-143 inhibits insulin-stimulated AKT activation and impairs glucose metabolism. Nat Cell Biol 2011;13:434-46.

22 Najafi-Shoushtari SH, Kristo F, Li Y, et al. MicroRNA-33 and the SREBP host genes cooperate to control cholesterol homeostasis. Science 2010;328:1566-9.

23 Niu Y, Lin Z, Wan A, et al. Rna N6-methyladenosine demethylase FTO promotes breast tumor progression through inhibiting BNIP3. Mol Cancer 2019;18:46.

24 The Cancer Genome Atlas Network. Comprehensive molecular characterization of human colon and rectal cancer. Nature 2012;487:330-7.

25 Agranat-Tamir L, Shomron N, Sperling J, et al. Interplay between pre-mRNA splicing and microRNA biogenesis within the supraspliceosome. Nucleic Acids Res 2014:42:4640-51.

26 Kucherenko MM, Shcherbata HR. miRNA targeting and alternative splicing in the stress response - events hosted by membrane-less compartments. J Cell Sci 2018;131:jcs202002.

27 Jurica MS, Licklider LJ, Gygi SP, et al. Purification and characterization of native spliceosomes suitable for three-dimensional structural analysis. RNA 2002:8:426-39.

28 Gregory RI, Yan K-ping, Amuthan G, et al. The Microprocessor complex mediates the genesis of microRNAs. Nature 2004;432:235-40.

29 Chiarugi A, Dölle C, Felici R, et al. The NAD metabolome - a key determinant of cancer cell biology. Nat Rev Cancer 2012;12:741-52.

30 Sabharwal SS, Schumacker PT. Mitochondrial ROS in cancer: initiators, amplifiers or an Achilles' heel? Nat Rev Cancer 2014;14:709-21.

31 Dominissini D, Moshitch-Moshkovitz S, Schwartz S, et al. Topology of the human and mouse M6a RNA methylomes revealed by m6A-seq. Nature 2012;485:201-6.

32 Alarcón CR, Lee H, Goodarzi H, et al. N6-Methyladenosine marks primary microRNAs for processing. Nature 2015;519:482-5.

33 Liu N, Dai Q, Zheng G, et al. N6-methyladenosine-dependent RNA structural switches regulate RNA-protein interactions. Nature 2015;518:560-4.

34 Alarcón CR, Goodarzi H, Lee $H$, et al. Hnrnpa2B1 is a mediator of m6A-Dependent nuclear RNA processing events. Cell 2015;162:1299-308.

35 Geuens T, Bouhy D, Timmerman V. The hnRNP family: insights into their role in health and disease. Hum Genet 2016:135:851-67.

36 Lu J, Getz G, Miska EA, et al. Microrna expression profiles classify human cancers. Nature 2005:435:834-8.

37 Kumar MS, Lu J, Mercer KL, et al. Impaired microRNA processing enhances cellular transformation and tumorigenesis. Nat Genet 2007;39:673-7.

38 Nguyen TA, Jo MH, Choi Y-G, et al. Functional anatomy of the human microprocessor. Cell 2015;161:1374-87.

39 Bajan S, Hutvagner G. Another "Loophole" in miRNA Processing. Mol Cell 2011:44:345-7.

40 Trabucchi M, Briata P, Garcia-Mayoral M, et al. The RNA-binding protein KSRP promotes the biogenesis of a subset of microRNAs. Nature 2009;459:1010-4. 
41 Kawahara Y, Mieda-Sato A. Tdp-43 promotes microRNA biogenesis as a component of the Drosha and Dicer complexes. Proc Natl Acad Sci U S A 2012;109:3347-52.

42 Han C, Liu Y, Wan G, et al. The RNA-binding protein DDX1 promotes primary microRNA maturation and inhibits ovarian tumor progression. Cell Rep 2014;8:1447-60.

43 Fan B, Sutandy FXR, Syu G-D, et al. Heterogeneous ribonucleoprotein K (hnRNP K) binds miR-122, a mature liver-specific microRNA required for hepatitis $C$ virus replication. Molecular \& Cellular Proteomics 2015;14:2878-86.

44 Guil S, Cáceres JF. The multifunctional RNA-binding protein hnRNP A1 is required for processing of miR-18a. Nat Struct Mol Biol 2007;14:591-6.

45 Köster T, Meyer K, Weinholdt C, et al. Regulation of pri-miRNA processing by the hnRNP-like protein AtGRP7 in Arabidopsis. Nucleic Acids Res 2014;42:9925-36.

46 Liu M, Roth A, Yu M, et al. The IGF2 intronic miR-483 selectively enhances transcription from IGF2 fetal promoters and enhances tumorigenesis. Genes Dev 2013;27:2543-8.

47 Loo JM, Scherl A, Nguyen A, et al. Extracellular metabolic energetics can promote cancer progression. Cell 2015;160:393-406.
48 Veronese A, Lupini L, Consiglio J, et al. Oncogenic role of miR-483-3p at the IGF2/483 locus. Cancer Res 2010;70:3140-9.

49 Barbieri I, Tzelepis K, Pandolfini L, et al. Promoter-Bound METTL3 maintains myeloid leukaemia by m6A-dependent translation control. Nature 2017;552:126-31.

50 Frye $\mathrm{M}$, Harada BT, Behm M, et al. Rna modifications modulate gene expression during development. Science 2018;361:1346-9.

51 Niu Y, Wan A, Lin Z, et al. N6-Methyladenosine modification: a novel pharmacological target for anti-cancer drug development. Acta Pharm Sin B 2018;8:833-43.

52 Lin Z, Niu Y, Wan A, et al. RNA m(6) A methylation regulates sorafenib resistance in liver cancer through FOXO3-mediated autophagy. Embo J 2020:e103181.

53 Wu B, Su S, Patil DP, et al. Molecular basis for the specific and multivariant recognitions of RNA substrates by human hnRNP A2/B1. Nat Commun 2018;9:420.

54 Tsofack SP, Garand C, Sereduk C, et al. Nono and raly proteins are required for YB-1 oxaliplatin induced resistance in colon adenocarcinoma cell lines. Mo/ Cancer 2011;10:145.

$55 \mathrm{Xu}$ J, Liu Y, Li Y, et al. Precise targeting of POLR2A as a therapeutic strategy for human triple negative breast cancer. Nat Nanotechnol 2019;14:388-97. 